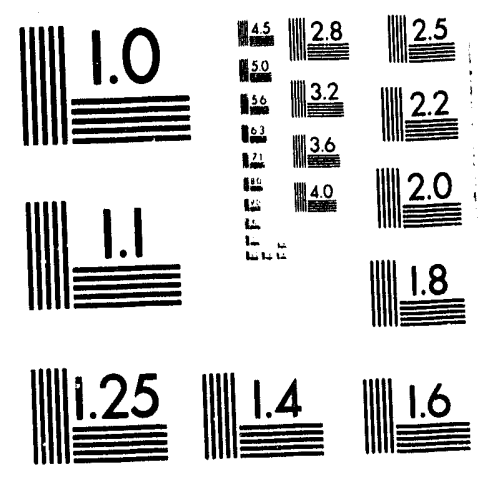



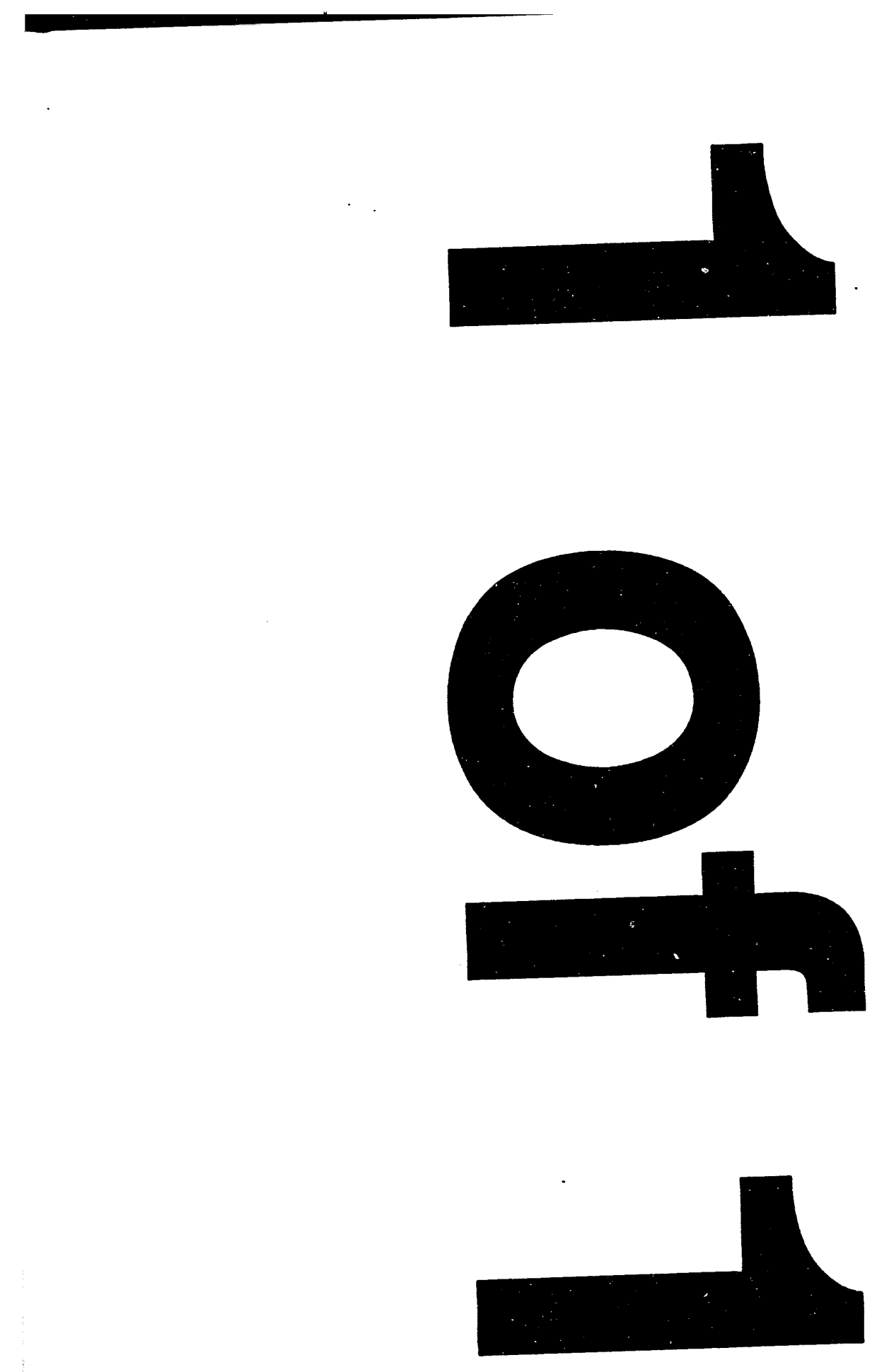
Distribution Category:

Mathematics and Computer Science (UC-405)

\author{
ARGONNE NATIONAL LABORATORY \\ 9700 South Cass Avenue \\ Argonne, Illinois 60439-4801
}

\title{
Otter 3.0 Reference Manual and Guide
}

by

\author{
William W. McCune
}

Mathematics and Computer Science Division

January 1994

This work was supported by the Ofice of Scientific Computing, U.S. Department of Energy, under Contract W-31-109-Eng-38. 


\section{Contents}

$\begin{array}{lr}\text { Abstract } & 1\end{array}$

1 Introduction 1

1.1 What OTter Isn't ................. 2

1.2 History, New Features, and Changes ............ 3

1.3 Useful Background . . . . . . . . . . . . . . . 4

2 Outline of Otter's Inference Process 4

3 Starting OTter $\quad 6$

4 Syntax $\quad 7$

4.1 Comments . . . . . . . . . . . . . . . 7

4.2 Names for Variables, Constants, Functions, and Predicates . . . . 7

4.3 Terms and Atoms . . . . . . . . . . . . . . 8

4.4 Literals and Clauses $\ldots \ldots \ldots \ldots \ldots$

4.5 Formulas $\ldots \ldots \ldots \ldots \ldots \ldots$

4.6 Infix, Prefix, and Postfix Expressions . . . . . . . . 10

4.7 Whitespace in Expressions . . . . . . . . . . . . . . . 12

4.8 Bugs, etc., in Input and Output of Expressions . . . . . . . . 13

4.9 Examples of Operator Declarations . . . . . . . . . . . . . 13

5 Commands and the Input File 14

5.1 Input of 0 tions $\ldots \ldots \ldots \ldots \ldots$

5.2 Input of Lists of Clauses . . . . . . . . . . . . . . 14

5.3 Input of Lists of Formulas . . . . . . . . . . . 15

5.4 Input of Lists of Weight Templates . . . . . . . . . . . . 15

5.5 The Commands lex, skolem, and Irpo_multiset_status . . . . . 16

5.6 Other Commands . . . . . . . . . . . . . . . . . 17

$\begin{array}{llr}6 & \text { Options } & 17\end{array}$

6.1 Flags . . . . . . . . . . . . . . . . . 17

6.1 .1 Main Loop Flags . . . . . . . . . . . . . 17 
6.1 .2 Inference Rules . . . . . . . . . . . . . 18

6.1 .3 Paramodulation Flags . . . . . . . . . . . . . . 19

6.1.4 Flags fo $\mathrm{f}_{4}$ Handling Generated Clauses . . . . . . . . . 19

6.1.5 Demodulation and Ordering Flags . . . . . . . . . 20

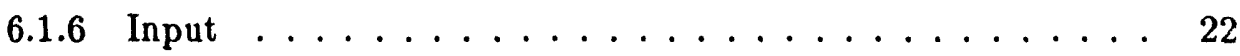

6.1 .7 Output Flags ...................... 23

6.1 .8 Indexing Flags $\ldots \ldots \ldots \ldots \ldots \ldots$

6.1 .9 Miscellaneous Flags $\ldots \ldots \ldots \ldots . \ldots \ldots$

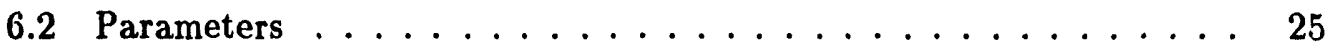

6.2.1 Monitoring Progress ................. 25

6.2.2 Placing Limits on the Search ............. 25

6.2.3 Limits on Properties of Generated Clauses . . . . . . . . . 25

6.2 .4 Indexing Parameters ................ 26

6.2.5 Miscellaneous Parameters .............. 26

$\begin{array}{llr}7 & \text { Demodulation } & 27\end{array}$

8 Ordering and Dynamic Demodulation 29

8.1 Ad Hoc Ordering . . . . . . . . . . . . . . . . 30

8.1 .1 Term Ordering (Ad Hoc) $\ldots \ldots \ldots \ldots$

8.1 .2 Orienting Equalities (Ad Hoc) $\ldots \ldots \ldots 31$

8.1.3 Determining Dynamic Demodulators (Ad Hoc) . . . . . . . 31

8.1.4 Lex-dependent Demodulation (Ad Hoc) . . . . . . . . 32

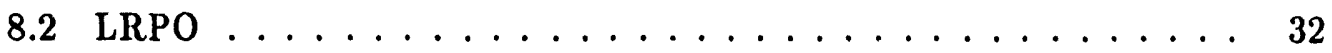

8.2 .1 Term Ordering (LRPO) . . . . . . . . . . . 32

8.2 .2 Orienting Equalities (LRPO) .............. 33

8.2.3 Determining Dynamic Demodulators (LRPO) . . . . . . 33

8.2.4 LRPO-dependent Demodulation (LRPO) . . . . . . . . 33

8.3 Knuth-Bendix Completion . . . . . . . . . . . . . . 33

9 Evaluable Functions and Predicates (\$SUM, \$LT, ...) 35

9.1 Using More Natural Expressions for Evaluation . . . . . . . . . . 38

9.2 Evaluation Examples . . . . . . . . . . . . . . 39 
10 Weighting $\quad 40$

10.1 Weighing Clauses and Literals . . . . . . . . . . . . 41

10.2 Weighing Atoms and Terms ............... 41

11 Answer Literals

12 The Passive List 42

13 Completeness and Soundness $\quad 42$

13.1 Completeness . . . . . . . . . . . . . . . . 42

13.2 Soundness ......................... 43

14 Interaction during the Search 43

15 Output and Exit Codes $\quad 44$

16 Controlling Memory $\quad 46$

17 Fringe Features $\quad 47$

17.1 Autonomous Mode . . . . . . . . . . . . . . . . 47

17.2 The Hot List $\ldots \ldots \ldots \ldots \ldots \ldots$

17.3 Linked UR-Resolution . . . . . . . . . . . . . . . . . 49

17.4 Conditional Demodulation . . . . . . . . . . . . . . . 49

17.5 Special Unary Function Demodulation . . . . . . . . . . . . 49

17.6 Ancestor Subsumption . . . . . . . . . . . . 50

17.7 Reducing max_weight on the Fly . . . . . . . . . . . 51

17.8 The Invisible Argument $\ldots \ldots \ldots \ldots$

17.9 Floating-Point Operations . . . . . . . . . . . . . . 51

17.10 Foreign Evaluable Functions . . . . . . . . . . . 52

17.11 Sequent Notation for Clauses . . . . . . . . . . . . 52

17.12 The Inference Rule $g L$ for Cubic Curves . . . . . . . . . . 53

18 Limits, Abnormal Ends, and Fixes $\quad 54$

19 Obtaining and Installing OTTER 55 
Acknowledgments

References 


\title{
OtTer 3.0 Reference Manual and Guide
}

\author{
by
}

William W. McCune

mccune@mcs.anl.gov

\begin{abstract}
OTter (Organized Techniques for Theorem-proving and Effective Research) is a resolution-style theorem-proving program for first-order logic with equality. OTTER includes the inference rules binary resolution, hyperresolution, UR-resolution, and binary paramodulation. Some of its other abilities and features are conversion from first-order formulas to clauses, forward and back subsumption, factoring, weighting, answer literals, term ordering, forward and back demodulation, evaluable functions and predicates, and Knuth-Bendix completion. OTTER is coded in $\mathrm{C}$, is free, and is portable to many different kinds of computer.
\end{abstract}

\section{Introduction}

OtTer (Organized Techniques for Theorem-proving and Effective Research) is a resolution-style theorem prover, similar in scope and purpose to the AURA [22] and LMA/ITP [15] theorem provers, which are also associated with Argonne. OTTER applies to statements written in first-order logic with equality. The primary design considerations have been performance, portability, and extensibility. The programming language $\mathrm{C}$ is used.

OTTER features the inference rules binary resolution, hyperresolution, URresolution, and binary paramodulation. These inference rules take a small set of clauses and infer a clause; if the inferred clause is new, interesting, and useful, it is stored and may become available for subsequent inferences.

Other features of OTTER are the following: 
- Statements of the problem may be input either with first-order formulas or with clauses (a clause is a disjunction with implicit universal quantifiers and no existential quantifiers). If first-order formulas are input, OTTER translates them to clauses.

- Forward demodulation rewrites and simplifies newly inferred clauses with a set of equalities, and back demodulation uses a newly inferred equality (which has been added to the set of demodulators) to rewrite all existing clauses.

- Forward subsumption deletes an inferred clause if it is subsumed by any existing clause, and back subsumption deletes all clauses that are subsumed by an inferred clause.

- A variant of the Knuth-Bendix method can search for a complete set of reductions and help with proof searches.

- Weight functions and lexical ordering decide the "goodness" of clauses and terms.

- Answer literals can give information about the proofs that are found.

- Evaluable functions and predicates build in integer arithmetic, Boolean operations, and lexical comparisons and enable users to "program" aspects of deduction processes.

Although OTTER has an autonomous mode, most work with OTTER involves interaction with the user. After the user has encoded a problem into first-order logic or into clauses, he or she usually chooses inference rules, sets options to control the processing of inferred clauses, and decides which input formulas or clauses are to be in the initial set of support and which (if any) equalities are to be demodulators. If OTTER fails to find a proof, the user may wish to try again with different initial conditions. In the autonomous mode, the user inputs a set of clauses and/or formulas, and OTTER does a simple syntactic analysis and decides inference rules and strategies. The autonomous mode is frequently useful for the first attempt at a proof.

\subsection{What OTTER Isn't}

Some of the first applications that come to mind when one hears "automated theorem proving" are number theory, calculus, and plane geometry, because these are some of the first areas in which math students try to prove theorems. Unfortunately, OTTER cannot do much in these areas: interesting number theory problems usually require induction, interesting calculus and analysis problems usually require higherorder functions, and the first-order axiomatizations of geometry are not practical. (Nonetheless, Art Quaife has proved many interesting theorems in number theory and geometry using OTTER $[20,19]$.) For practical theorem proving in inductive theories, see the work of Boyer and Moore $[2,3]$.

OTTER is also not targeted toward synthesizing or verifying formal hardware or software systems. See $[7,6]$ for work in those areas. 
Summaries of other theorem-proving systems can be found in proceedings of the recent Conferences on Automated Deduction (CADE) [23, 9].

\subsection{History, New Features, and Changes}

There have been several previous releases of OTTER-version 0.9 was distributed at CADE-9 in May 1988, version 1.0 was released in January 1989, version 2.0 in March 1990, and version 2.2 July 1991 . There was also a minor release, version 2.2ax, in January 1992 .

\section{Summary of New Features}

- In the autonomous mode (Sec. 17.1), the user simply inputs clauses and/or formulas, and OTTER decides on inference rules and strategies.

- The hot list (Sec. 17.2) can be used to give emphasis to some of the input clauses. (Suggested by Larry Wos.)

- The user can declare function symbols to be infix with associativity and precedence so that expressions can be written in a natural way (Sec. 4.6).

- Clauses can be written in sequent notation (Sec. 17.11). (Suggested by Art Quaife.)

- The new evaluable functions include bit string operations (Sec. 9) and floatingpoint operations (Sec. 17.9).

- The inference rule $g L$ builds in a generalization principle for cubic curves (Sec. 17.12). (Suggested by R. Padmanabhan.)

- The user can write in C his or her own evaluable operations (Sec. 17.10).

- Given clauses can be selected interactively (Sec. 6.1.1). (Suggested by Bob Veroff.)

- Ordered hyperresolution (Sec. 6.1.9) has been implemented (suggested by Mark Stickel) and is the default.

- Some optimizations have been implemented for propositional problems (Sec. 6.1.9).

- The justification lists for binary resolvents and paramodulants now tell which literals or terms were unified to produce the clause (Sec 15).

\section{Summary of Changes}

- Term ordering has been simplified (Sec. 8).

- Factoring is now applied also as a simplification rule (Sec. 6.1.4); for example, the clause $p(x) \mid p(a)$ simplifies to $p(a)$. 
- Conditional demodulators (Sec. 17.4) are written in a more natural way.

- Some of the options cause other options to be changed automatically. The automatically changed options can now be overridden.

- Setting the flag binary_res causes the flags factor and unit_deletion to be automatically set.

- The flag knuth_bendix causes a different set of options to be changed (Secs. 6.1.5 and 8.3).

- Multipliers in weight templates are written differently (Sec. 10).

- The parameter value that indicates "no limit" or "no action" has been changed from 0 to -1 for the parameters max_seconds, max_mem, max given, max $_{-} g e n$, max_kept, max_literals, max_proofs, demod_limit, and report.

Bugs in OtTer 2.2. The following bugs in OTter 2.2 have been fixed.

- Calculation of the level of a proof would sometimes cause OTTER 2.2 to hang just after printing the --- PROOF --- message.

- In some cases, OtTer 2.2 would crash when doing complicated demodulation during evaluation of a negative evaluable literal during hyperresolution.

- Unit deletion with a unit clause containing an answer literal with variables not in the ordinary literal was not handled correctly.

- When a multiliteral clause merged into an equality unit and the flag dynamic_demod was set, the equality would never become a demodulator.

\subsection{Useful Background}

This manual does not contain an introduction to first-order logic or to automated deduction. We assume that the reader knows the basic terminology including term (variable, constant, complex term), atom, literal, clause, propositional variable, function symbol, predicate symbol, Skolem constant, Skolem function, formula, and conjunctive normal form $(C N F)$, resolution, hyperresolution, and paramodulation. See [25], [4], or [14] for an introduction to automated theorem proving, see [26] for an overview of the field, see [21] and [1] for collections of important papers, and see [24] for a list of outstanding general problems in the field.

\section{Outline of OtTer's Inference Process}

Once OTTER gets going with its real work-making inferences and searching for proofs-it operates on clauses and on clauses only. If the user inputs nonclausal first-order formulas, OTTER immediately generates clauses from them. 
As with its predecessors AURA and LMA/ITP, OTTER's basic inference mechanism is the given-clause algorithm, which can be viewed as a simple implementation of the set of suppcrt strategy [25]. OTTER maintains four lists of clauses:

usable. This list contains clauses that are available to make inferences.

sos. Clauses in list sos (set of support) are not available to make inferences; they are waiting to participate in the search.

passive. These clauses do not directly participate in the search; they are used only for forward subsumption and unit conflict. The passive list is fixed at input and does not change during the search. See Sec. 12.

demodulators. These are equalities that are used as rules to rewrite newly inferred clauses.

The main loop for inferring and processing clauses and searching for a refutation operates mainly on the lists usable and sos:

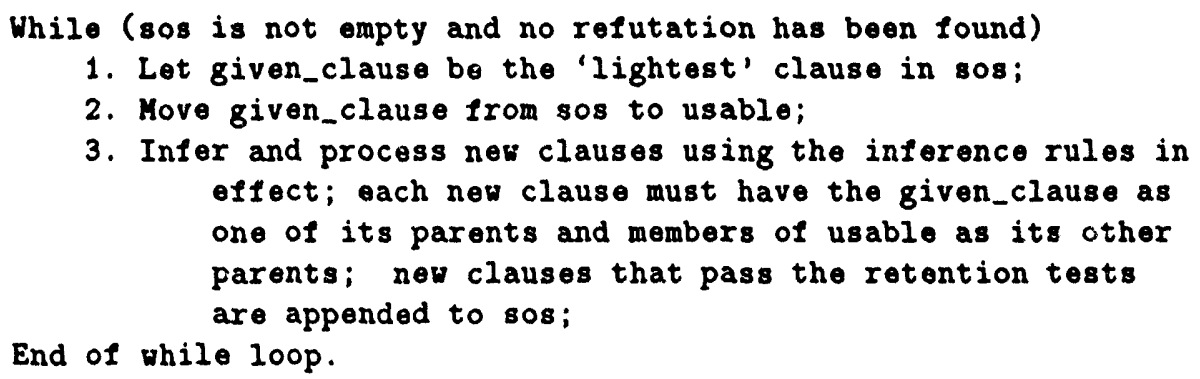

The set of support strategy requires the user to partition the input clauses into two sets: those with support and those without. For each inference, at least one of the parents must have support. Retained inferences receive support. In other words, no inferences are made in which all parents are nonsupported input clauses. At input time, O'TTER's list sos is the set of supported clauses, and usable is the nonsupported clauses. (Once the main loop has started, usable no longer corresponds to nonsupported clauses, because sos clauses have moved there.) OTTER's main loop implements the set of support strategy, because no inferences are made in which all of the parents are from the initial usable list.

The following paragraph tries to answer the frequently asked question "At a certain point, OTTER has all of the clauses available to make the inference I want, and one of the potential parents is selected as the given clause-why doesn't the program make the inference?"

OTTER's main loop eliminates an important kind of redundancy. Suppose one can infer clause $C$ from clauses $A$ and $B$, and suppose both $A$ and $B$ are in list sos. If $A$ is selected as the given clause, it will be moved to usable and inferences will be made; but $A$ will not mate with $B$ to infer $C$, because $B$ is still in sos. We must wait until $B$ has also been sclected as given clause. Otherwise, we would infer $C$ twice. (The redundancy would be much worse with inference rules such 
as hyperresolution and UR-resolution that can use many parents.) In general, all parents that participate in an inference must either have been in the initial usable list or have been selected as given clauses. (This is not true when demodulators are considered as parents.)

The procedure for processing a newly inferred clause new_cl follows; steps marked with * are optional.

1. Renumber variables.

* 2. Output new_cl.

3. Demodulate new_cl (including \$ evaluation).

* 4. Orient equalities.

* 5. Apply unit deletion.

6. Merge identical literals (leftmost copy is kept).

* 7. Apply factor-simplification.

* 8. Discard new_cl and exit if nen_cl has too many literals or variables.

9. Discard new_cl and exit if nen_cl is a tautology.

* 10. Discard new_cl and exit if new_cl is too 'heavy'.

* 11. Sort literals.

* 12. Discard new_cl and exit if new_cl is subsumed by any clause in usable, sos, or passive (forward subsumption).

13. Integrate new_cl and append it to sos.

* 14. Output kept clause.

15. If new_cl has 0 literals, a refutation has been found.

16. If new_cl has 1 literal, then search usable, sos, and passive for unit conflict (refutation) with ner_cl.

* 17. Print the proof if a refutation has been Iound.

* 18. Try to make new_cl into a demodulator.

* 19. Back demodulate if Step 18 made new_cl into a demodulator.

* 20. Discard each clause in usable or 808 that is subsumed by non_cl (back subsumption).

* 21. Factor nen_cl and process factors.

Steps 19-21 are delayed until steps 1-18 have been applied to all clauses inferred from the active given clause.

\section{Starting OTtER}

Although OTTER has a primitive interactive feature (Sec. 14), it is essentially a noninteractive program. On UNIX-like systems it reads from the standard input and writes to the standard output:

otter < input-file > output-file

No command-line options are accepted; all options are given in the input file. 


\section{Syntax}

OTTER recognizes two basic types of statement: clauses and formulas. Clauses are simple disjunctions whose variables are implicitly universally quantified. OTTER's searches for proofs operate on clauses. Formulas are first-order statements without free variables-all variables are explicitly quantified. When formulas are input, OTTER immediately translates them to clauses.

Function symbols and predicate symbols are sometimes referred to as functors when the distinction is not important.

\subsection{Comments}

Comments can be placed in the input file by using the symbol \%. All characters from the first $\%$ on a line to the end of the line are ignored. Comments can occur within terms. Comments are not echoed to the output file.

\subsection{Names for Variables, Constants, Functions, and Predicates}

Three kinds of character string, collectively referred to as names, can be used for variables, constants, function symbols, and predicate symbols:

- An ordinary name is a string of alphar umerics, \$, and ..

- A special name is a string of characters in the set $*+-\Lambda^{-}<>z^{\prime *}: ? \bullet 2 ! ; *$ (and sometimes ().

- A quoted name is any string enclosed in two quotation marks of the same type, either " or '. We have no trick for including a quotation mark of the same type in a quoted name.

(The reason for separating ordinary and special names has to do with infix, prefix, and postfix operators; see Sec. 4.6.) Although out of place here, for completeness we list the meanings of the remaining printable characters.

- . (period) - terninates input expressions.

- \% - starts a comment (which ends with the end of the line).

- , () []\{\} (and sometimes 1) - are punctuation and grouping symbols.

Variables. Determining whether a simple term is a constant or a variable depends on the context of the term. If it occurs in a clause, the symbol determines the type: the default rule is that a simple term is a variable if it starts with $u, v, w, x, y$, or z. If the flag prolog_style_variables is set, a simple term is a variable if and only if it starts with an upper-case letter or with .. (Therefore, variables in clauses must be ordinary names.) A simple term in a formula is a variable if and only if it is bound by a quantifier. 
Reserved and Built-in Names. Names that start with $\$$ are reserved for special purposes, including evaluable functions and predicates (Sec. 9), answer literals and terms (Sec. 11), and some internal system names. The name $=$ and any name that starts with eq, EQ, or Eq, when used as a binary predicate symbol, is recognized as an equality predicate by the demodulation and paramodulation processes. And some names, when they occur in clauses or formulas, are recognized as logic symbols.

Overloaded Symbols. The user can use a name for more than one purpose, for example as a constant and as a 5-ary predicate symbol. When the flag check_arity is set (the default), the user is warned about such uses. Some built-in names are also overloaded; for example, $I$ is used both for disjunction and as Prolog-style list punctuation, and although - is built in as logical negation, it is generally used for both unary and binary minus as well.

\subsection{Terms and Atoms}

Recall that, when interpreted, terms are evaluated as objects in some domain, and atoms are evaluated as truth values. Constants and variables are terms. An $n$ ary function symbol applied to $n$ terms is also a term. An $n$-ary predicate symbol applied to $n$ terms is an atom. A nullary predicate symbol (also referred to as a propositional variable) is also an atom.

The pure way of writing complex terms and atoms is with standard application: the function or predicate symbol, opening parenthesis, arguments separated by commas, then closing parenthesis, for example, $f(a, b, c)$ and $=(f(x, e), x)$. If all subterms of a term are written with standard application, the term is in pure prefix form. Whitespace (spaces, tabs, newlines, and comments) can appear in standard application terms anywhere except between a function or predicate symbol and its opening parenthesis. If the flag display_terms is set, OTTER will output terms in pure prefix form.

Infix Equality. Some binary functors can be written in infix form; the most important is $=$. In addition, a negated equality, $-(a=b)$ can be abbreviated $a !=b$.

List Notation. Prolog-style list notation can be used to write terms that represent lists. Table 1 gives some example terms in list notation and the corresponding pure prefix form. Of course, lists can contain complex terms, including other lists.

\begin{tabular}{ll}
\multicolumn{2}{c}{ Table 1: List Notation } \\
\hline[] & $\$ n i l$ \\
{$[x \mid y]$} & $\$ \operatorname{cons}(x, y)$ \\
{$[x, y]$} & $\$ \operatorname{cons}(x, \$ \operatorname{cons}(y, \$ n i l))$ \\
{$[a, b, c, d]$} & $\$ \operatorname{cons}(a, \$ \operatorname{cons}(b, \$ \operatorname{cons}(c, \$ \operatorname{cons}(d, \$ n i l))))$ \\
{$[a, b, c \mid x]$} & $\$ \operatorname{cons}(a, \$ \operatorname{cons}(b, \$ \operatorname{cons}(c, x)))$ \\
\hline
\end{tabular}




\subsection{Literals and Clauses}

A literal is either an atom or the negation of an atom. A clause is a disjunction of literals. The built-in symbols for negation and disjunction are - and I, respectively. Although clauses can be written in pure prefix form, with - as a unary symbol and $I$ as a binary symbol, they are rarely written that way. Instead, they are almost always written in infix form, without parentheses. For example, the following is a clause in both forms.

Pure prefix: $\quad \mid(-(a), \mid(=(b 1, b 2),-(=(c 1, c 2))))$

Infix (abbreviated): $\quad-a|b 1=b 2 \quad| \quad c 1 !=c 2$

Otter accepts both forms. (Clauses are parsed by the general term-parsing mechanism presented in Sec. 4.6).

\subsection{Formulas}

Table 2 lists the built-in logic symbols for constructing formulas.

Table 2: Logic Symbols

\begin{tabular}{ll}
\hline negation & - \\
disjunction & 1 \\
conjunction & 2 \\
implication & $->$ \\
equivalence & $\langle->$ \\
existential quantification & exists \\
universal quantification & all \\
\hline
\end{tabular}

Formulas in Pure Prefix Form. Although the practice is rarely done, formulas can be written in pure prefix form. Quantification is the only tricky part: there is a special variable-arity functor, \$Quantified, for quantified formulas. For example, $\forall x y \exists z(P(x, y, z) \mid Q(x, z))$ is represented by

$$
\$ Q \text { uantified(all, } x, y, \text { exists, } z, I(P(x, y, z), Q(x, z))) \text {. }
$$

If the flag display_terms is set, the formulas (and everything else) will be displayed in pure prefix form.

Abbreviated Formulas. Formulas are usually abbreviated in a natural way. The associativity and precedence rules for abbreviating formulas and the mechanism for parsing formulas are presented in Sec. 4.6. Here are some examples.

standard usage

$\forall x P(x)$
OTTER syntax (abbreviated)

all $x P(x)$ 


$$
\begin{array}{ll}
\forall x y \exists z(P(x, y, z) \vee Q(x, z)) & \text { all } x \text { y exists } z(\mathrm{P}(\mathrm{x}, \mathrm{y}, \mathrm{z}) \mid \mathrm{Q}(\mathrm{x}, \mathrm{z})) \\
\forall x(P(x) \wedge Q(x) \wedge R(x) \rightarrow S(x)) & \text { all } \mathrm{x}(\mathrm{P}(\mathrm{x}) \& \mathrm{Q}(\mathrm{x}) \& \mathrm{R}(\mathrm{x}) \rightarrow \mathrm{S}(\mathrm{x}))
\end{array}
$$

Note that if a formula has a string of identical quantifiers, all but the first can be dropped. For example, all $x$ all $y$ all $z p(x, y, z)$ can be shortened to all $x$ y $z p(x, y, z)$. In expressions involving the associative operations \& and I, extra parentheses can be dropped. Moreover, a default precedence on the logic symbols allows us to drop more parentheses: $\langle-\rangle$ has the same precedence as $\rightarrow$, and the rest in decreasing order are $\rightarrow, 1, \&,-$. Greater precedence means closer to the root of the term (i.e., larger scope). For example, $p|-q \& r \rightarrow-s| t$ represents $(p \mid(-(q) \& r)) \rightarrow(-(s) \mid t)$, or in pure prefix form, $\rightarrow(|(p, \&(-(q), r))|,(-(s), t))$.

When in doubt about how a particular string will be parsed, one can simply add additional parentheses and/or test the string by having OTTER read it and then display it in pure prefix form. The following input file can be used to test the preceding example.

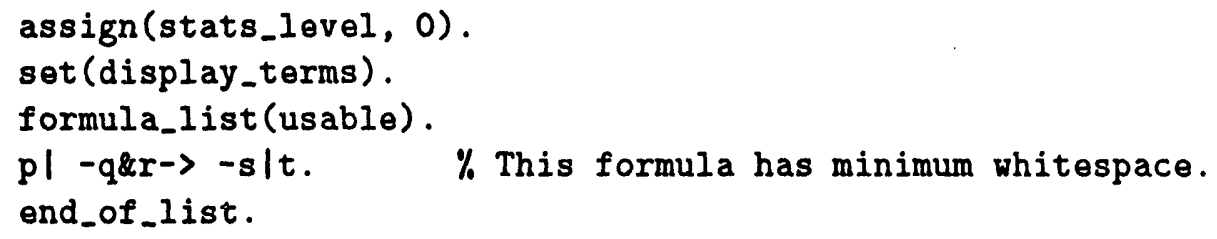

In general, $u$ hitespace is required around 211 and exists and to the left of -; otherwise, whitespace around the logic symbols can be removed. See Sec. 4.6 for the rules.

\subsection{Infix, Prefix, and Postfix Expressions}

Many Prolog systems (for example Quintus and Sicstus) have a feature that allows users to declare that particular function or predicate symbols are infix, prefix, or postfix and to specify a precedence and associativity so that parentheses can sometimes be dropped. OTTER has a similar feature. In fact, the clause and formula parsing routines use the feature. Users who use only the predeclared logic operators for clauses and formulas and the predeclared infix equality $=$ can skip the rest of this section.

Prolog users who are familiar with the declaration mechanism should note the following differences between the Quintus/Sicstus mechanism and OTTER's.

- The predeclared operators are different. See Table 3.

- OtTer does not treat comma as an operator; in particular, $a, b, c$ cannot be a term, as in $a, b, c \rightarrow d, \theta, f$.

- OTter treats the quantificrs all and exists as special cases, because they don't seem to fit neatly into the standard Prolog mechanism. 
- OtTer requires whitespace in some cases where the Prologs do not.

Functors to be treated in this special way are given a type and a precedence. Either OTTER predeclares the functor's properties, or the user gives OTTER a command of one of the forins

op (precedence, type, functor).

op (precedence, type, list-of-functors).

The precedence is an integer $i, 0<i<1000$, and type is one of the following: $x f x$, $x f y, y f x$ (infix), $f x, f y$ (prefix), $x f, y f$ (postfix). See Table 3 for the commands corresponding to the predeclared functors.

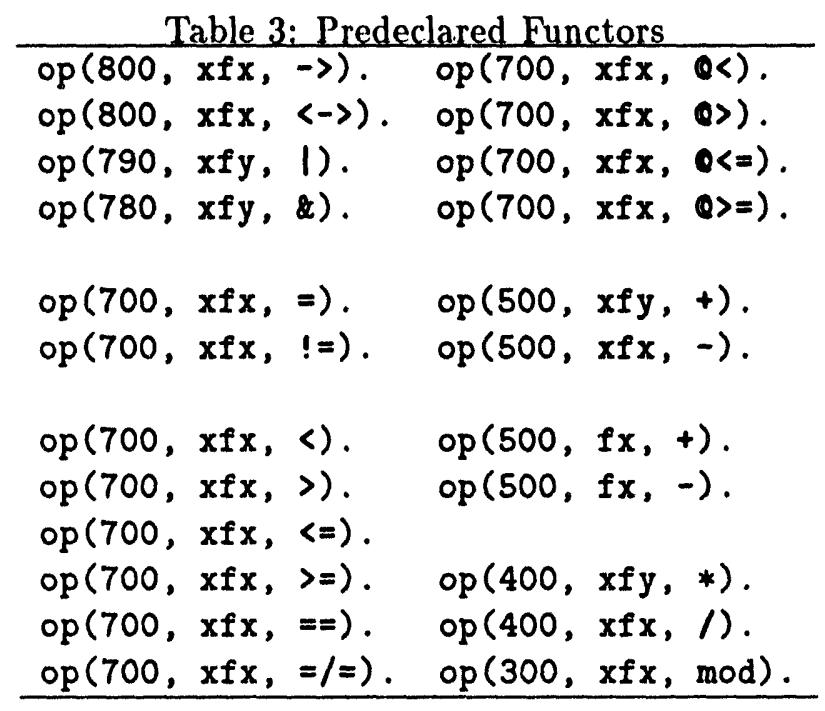

Given an expression that looks like it might be associated in a number of ways, the relative precedence of the operators determines, in part, how it is associated. A functor with higher precedence is more dominant (closer to the root of the term), and one with lower precedence binds more tightly. For example, the functors $\rightarrow, 1$, $\&$, and - have decreasing precedence; therefore the expression $p \&-q \mid r \rightarrow s$ is understood as $((p \&(-q)) \mid r) \rightarrow s$.

In each of the types, $f$ represents the functor, and $x$ and $y$, which represent the expressions to which the functor applies, specify how terms are associated. Given an expression involving functors of the same precedence, the types of the functors determines, in part, the association. See Table 4. The following are examples of associativity:

- If + has type $x f y$, then $a+b+c+d$ is understood as $a+(b+(c+d))$.

- If $\rightarrow$ has type $x f x$, then $a->b->c$ is not well formed.

- If - has type $f y$, then - - $p$ is understood as $-(-(-(p)))$. (The spaces are necessary; otherwise, --- will be parsed as single name.) 


\section{Table 4: Functor Types}

\begin{tabular}{lll}
\hline $\mathbf{x f x}$ & infix (binary) & don't associate \\
$\mathbf{x f y}$ & infix (binary) & associate right \\
$\mathbf{y} \mathbf{x}$ & infix (binary) & associate left \\
$\mathbf{f x}$ & prefix (unary) & don't associate \\
$\mathbf{f y}$ & prefix (unary) & associate \\
$\mathbf{x f}$ & postfix (unary) & don't associate \\
$\mathbf{y x}$ & postfix (unary) & associate \\
\hline
\end{tabular}

- If - has type $\mathbf{f x}$, then - - -p is not well formed.

Caution: The associativity specifications in the infix functor declarations say nothing about the logical associativity of the operation, e.g., whether $(a+b)+c$ is the same object as as $a+(b+c)$. The specifications are only about parsing ambiguous expressions. In most cases, when an operator is $\mathbf{x f y}$ or $\mathbf{y f x}$, it is also logically associative, but the logical associativity is handled separately; it is built-in in the case of the logic symbols $I$ and \& in OTTER clauses and formulas, and it must be axiomatized in other cases.

Details of the Functor Declarations. (This paragraph can be skipped by most users.) The precedence of functors extends to the precedence of expressions in the following way. The precedence of an atomic, parenthesized, or standard application expression is 0 . Respective examples are $p,(x+y)$, and $p(a+b, c, d)$. The precedence of a (well-formed) nonparenthesized nonatomic expression is the same as the precedence of the root functor. For example, a\&b has the precedence of $\&$, and a\&b/c has the precedence of the greater functor. In the type specifications, $x$ represents an expression of lower precedence than the functor, and y represents an expression with precedence less than or equal to the functor. Consider $a+b+c$, where + has type $x f y$; if association is to the left, then the second occurrence of + does not fit the type, because $a+b$, which corresponds to $x$, does not have a lower precedence than + ; if association is to the right, then all is well. If we extend the example, under the declarations op $(700, x f x, \Rightarrow)$ and op $(500, x f y,+)$, the expression $a+b+c=d+\theta$ must be understood as $(a+(b+c))=(d+\theta)$.

\subsection{Whitespace in Expressions}

The reason for separating ordinary names from special names (Sec. 4.2) is so that some whitespace (spaces, tabs, newline, and comments) can be removed. We can write $a+b+c$ (instead of having to write $a+b+c$ ), because " $a+b+c$ " cannot be a name, that is, it must be parsed into five names.

Caution. There is a deficiency in OTTER's parser having to do with whitespace between a name and opening parenthesis. The rule to use is: Insert some white space if and only if it is not a standard application. For example, the two picces of white space in $(a+(b+c))=(d+\theta)$ are required, and no white space is allowed after $f$ or $g$ in $f(x, g(x))$. 


\subsection{Bugs, etc., in Input and Output of Expressions}

- The symbol $I$ is either Prolog-style list punctuation or part of a special name. With the built-in declaration of $I$ as infix, the term [a/b] is ambiguous, with possible interpretations $t_{1}=\$ \operatorname{cons}(a, b)$ and $t_{2}=\$ \operatorname{cons}(I(a, b), \$ n i l)$. OTTER recognizes $[a \mid b]$ as $t_{1}$. The term $t_{2}$ can be written $[(a \mid b)]$. The bug is that $t_{2}$ will be output without the parentheses. This is the only case I know in which OTTER cannot correctly read a term it has written.

- A term consisting of a unary + or - applied to a nonnegative integer is always translated to a constant.

- Parsing large terms without parentheses, say $a 1+a 2+a 3+\ldots+a 1000$, can be very slow if the operator is left associative $(y f x)$. If you intend to parse such terms, make the operator right associative (xyf).

- Quoted strings cannot contain a quotation mark of the same type.

- The flag check_arity sometimes issues warnings when it should not.

- Braces $(\{\})$ can be used to group input expressions, but OTTER always uses ordinary parentheses on output.

\subsection{Examples of Operator Declarations}

Group Theory. Suppose we like to see group theory expressions in the form $\left(a b^{-1} c^{-1-1}\right)^{-1}$, in which right association is assumed. We can approximate this for OTTER with ( $\left.\left.a * b^{-} * c^{-}\right)^{-}\right)^{-}$. (We have to make the group operator explicit; -1 is not a legal OTTER name; the whitespace shown is required.) The declarations op $(400, x f y, *)$ and op (350, yf, $\left.{ }^{-}\right)$suffice. Other examples of expressions (with minimum whitespace) using these declarations are $(x * y) * z=x * y * z$ and $(y * x)^{-}=x^{-} * y^{-}$.

OTter Options. Options are normally input (Sec. 5.1) as in the following examples.

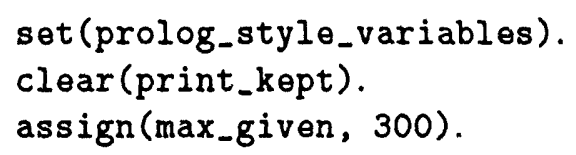

If, however, we make the declarations (the precedences are irrelevant)

$$
\begin{aligned}
& o p(100, f x, \text { set }) . \\
& o p(100, f x, \text { clear }) . \\
& o p(100, x f x \text {, assign). }
\end{aligned}
$$

then we may write 


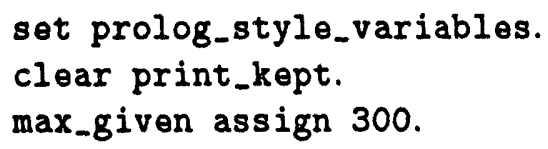

\section{Commands and the Input File}

Input to OTTER consists of a small set of commands, some of which indicate that a list of objects (clauses, formulas, or weight templates) follows the command. All lists of objects are terminated with end_of_list. The commands are given in Table 5. There are a few other commands for fringe features (Sec. 17).

\begin{tabular}{|c|c|}
\hline $\begin{array}{l}\text { include(file_name). } \\
\text { op(precedence, type, name(s)). } \\
\text { make_evaluable(sym, eval-sym). } \\
\text { set (flag_name). } \\
\text { clear(flag_name). } \\
\text { assign(parameter_name,integer). } \\
\text { list(list_name). } \\
\text { formula_list (list_name). } \\
\text { weight_ist (weight_list_name). } \\
\text { lex(symbol_list). } \\
\text { skolem(symbol_list). } \\
\text { lrpo_multiset_status (symbol_list). }\end{array}$ & $\begin{array}{l}\text { \% read input from another file } \\
\% \text { declare operator(s) } \\
\% \text { make a symbol evaluable } \\
\% \text { set a flag } \\
\% \text { clear a flag } \\
\% \text { assign an integer to a parameter } \\
\% \text { read a list of clauses } \\
\% \text { read a list formulas } \\
\% \text { read weight templates } \\
\% \text { assign an ordering on symbols } \\
\% \text { identify skolem functions } \\
\% \text { status for LRPo }\end{array}$ \\
\hline
\end{tabular}

\subsection{Input of Options}

OTTER recognizes two kinds of option: flags and parameters. Flags are Booleanvalued options; they are changed with the set and the clear commands, which take the name of the flag as the argument. Parameters are integer-valued options; they are changed with the assign command, which takes the name of the parameter as the first argument and an integer as the second. Examples are

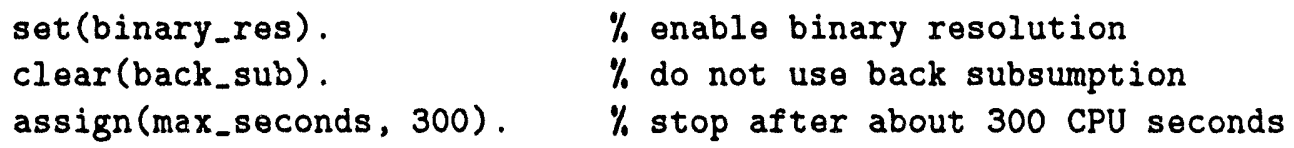

The options are described and their default values are given in Sec. 6 .

\subsection{Input of Lists of Clauses}

A list of clauses is specified with one of the following and is terminated with end_of_list. Each clause is terminated with a period. 


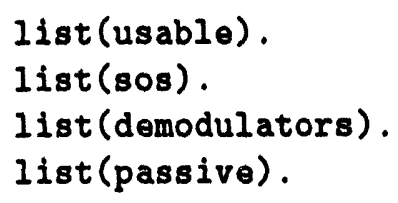

Example:

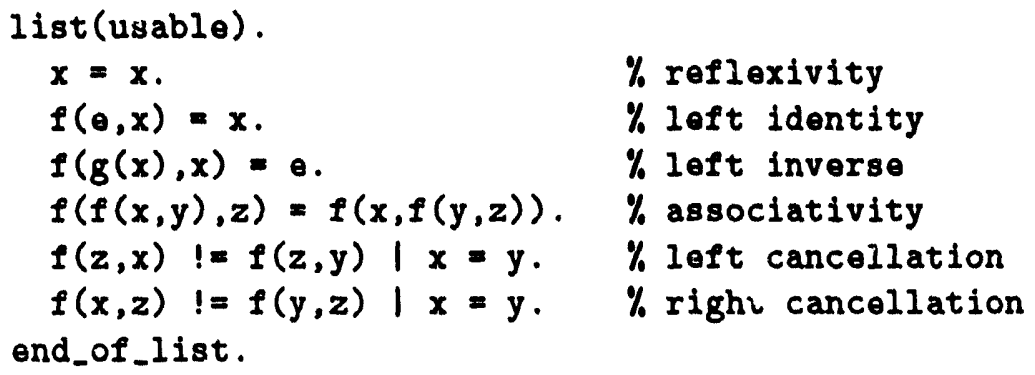

If the input contains more than one clause list of the same type, the lists will simply be concatenated.

\subsection{Input of Lists of Formulas}

A list of formulas is specified with one of the following and is terminated with end_of_list. Each formula is terminated with a period. (Note that demodulators cannot be input as formulas.)

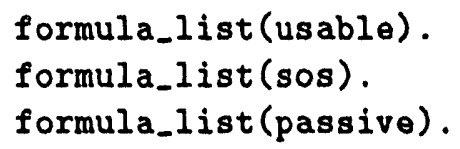

Example (analogous to above):

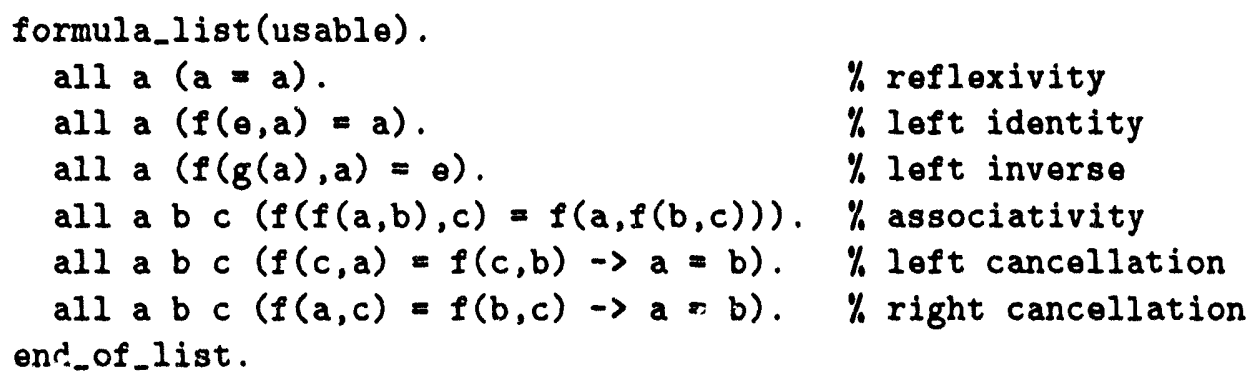

If the input contains more than one formula list of the same type, the lists will simply be concatenated.

\subsection{Input of Lists of Weight Templates}

A list of weight templates is specified with one of the following and is terminated with end_of_list. Each weight template is terminated with a period. 


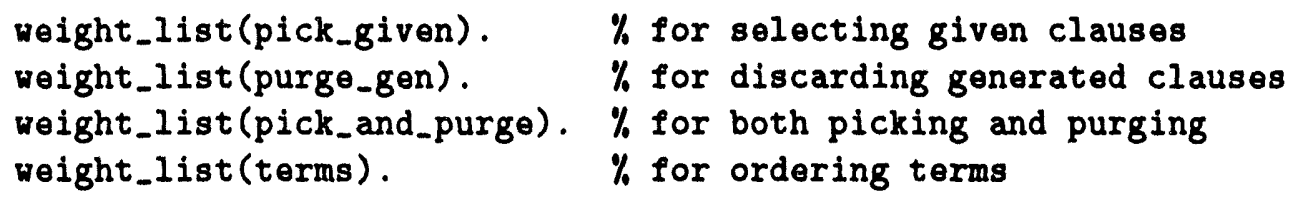

Example:

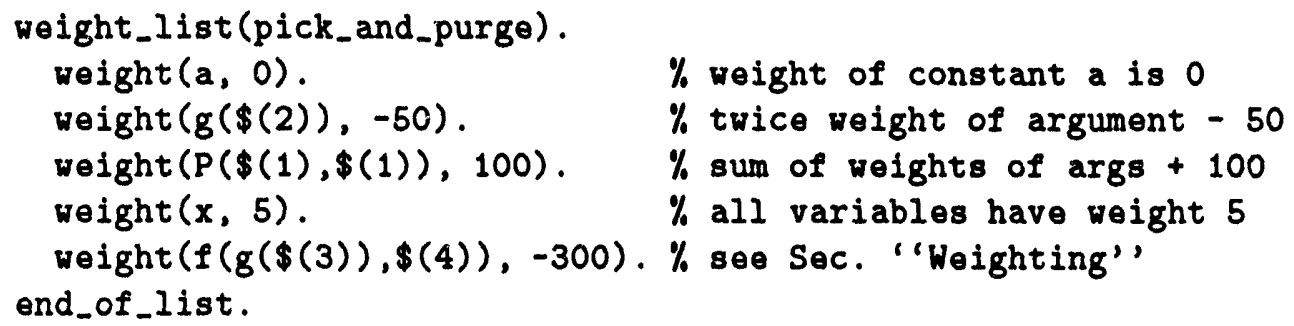

See Sec. 10 for the syntax and use of weight templates.

\subsection{The Commands lex, skolem, and lrpo_multiset_status}

Each of the commands lex, skolem, and 1rpo_multiset_status takes a list of terms as an argument. The lex command specifies an ordering on symbols, and the others give properties to symbols. An example is

$\operatorname{lex}\left(\left[a, b, f(\ldots), d,, g\left({ }_{-}\right), c\right]\right)$.

The arguments of $f$ and $g$ serve as place-holders only; they identify $f$ and $g$ as function or predicate symbols and specify the arity.

$\operatorname{lex}([\ldots])$. The lex command specifies an ordering (smallest-first) on function and constant symbols. Lexical ordering on terms is used in four contexts: orienting equality literals (Secs. 8.1.2 and 8.2.2), deciding whether an equality will be used as a demodulator (Secs. 8.1.3 and 8.2.3), deciding whether to apply a lex-dependent demodulator (Secs. 8.1.4 and 8.2.4), and evaluating functions/predicates that perform lexical comparisons (Sec. 9). If a lex command is not present, then OTTER uses a default ordering (Sec. 8).

skolem $([\ldots])$. The skolem command identifies constant and function symbols as Skolem symbols. (If the user inputs quantified formulas and OTTER Skolemizes, this command is not necessary.) The Skolem property is used by the options para_skip_skolem (Sec. 6.1.3) and delete_identical_nested_skolem (Sec. 6.1.4).

Irpo_multiset_status ([...]). This command specifies multiset status for the lexicographic recursive path ordering (flag Irpo). See Sec. 8.2. 


\subsection{Other Commands}

The command op (precedence, type, name (s)), example op $\left(400, x f y_{0}+\right)$, declares one or more symbols to have special properties with respect to input and output. See Sec. 4.6.

The command make_evaluable(symbol, evaluable-symbol), for example make_evaluable(_+_, $\$ \operatorname{SUM}\left({ }_{-},\right)_{-}$, copies evaluation properties from an evaluable symbol to another symbol, so that one can write $x+3$ instead of $\$ \operatorname{SUM}(x, 3)$. See Sec. 9.1.

The command include(file_name) causes input to be read from another input file. When the included file has been read, OTTER resumes reading commands after the include command. The file name must be recognized as an OTTER name, so if it contains characters such as period, slash, or hyphen, it must be enclosed in (single or double) quotes. Included files can include still other files. $A$ list of objects (clauses, formulas, or weight templates) cannot be split among different input files. One can, however, read clauses into a list from more than one file, as in the following example.

\begin{tabular}{l|l|l} 
standard input & file f1.in & file f2.in \\
\hline include("f1.in"). & list(usable). & list(usable). \\
include("f2.in"). & $\begin{array}{l}p(a) . \\
\text { end_of_list. }\end{array}$ & $\begin{array}{l}p(b) . \\
\text { end_of_list. }\end{array}$
\end{tabular}

\section{Options}

Flags are Boolean-valued options, and parameters are integer-valued options. When the user changes an option, OTTER sometimes automatically changes other options. The user is informed in the output file when such a change occurs.

Several additional flags and parameters are described in Sec. 17.

\subsection{Flags}

\subsubsection{Main Loop Flags}

A given clause is taken from sos at the beginning of each iteration of the main loop. The default is to take the lightest clause with respect to either weight_list (pick_given) or weight_list (pick_and_purge). If neither weight list is present, the weight of a clause is its number of symbols.

sos-queue - default clear. If this flag is set, the first clause in sos becomes the given clause (the set of support list operates as a queue). This causes a breadth-first search, also called level saturation. Some information about search levels is printed (see Sec. 15) when this flag is set.

sos_stack - default clear. If this flag is set, the last clause in sos becomes the 
given clause (the set of support list operates as a stack). This causes a depth-first search (which is almost never useful with OTTER).

input_sos_first - default clear. If this flag is set, the input clauses in sos are given a very low pick_given weight so that they are the first clauses selected as given clauses.

interactive_given - default clear. If this flag is set, then when it's time to select a new given clause, the user is prompted for his or her choice. This flag has priority over all other flags that govern selection of the given clause.

print_given - default set. If this flag is set, clauses are output when they become given clauses.

print_lists_at_end - default clear. If this flag is set, then usable, sos, and demodulators are printed at the end of the search.

\subsubsection{Inference Rules}

binary_res - default clear. If this flag is set, the inference rule binary resolution (along with any other inference rules that are set) is used to generate new clauses. Setting this flag causes the flags factor and unit_deletion to be automatically set.

hyper_res - default clear. If this flag is set, the inference rule (positive) hyperresolution (along with any other inference rules that are set) is used to generate new clauses.

neg_hyper_res - default clear. If this flag is set, the inference rule negative hyperresolution (along with any other inference rules that are set) is used to generate new clauses.

ur_res - default clear. If this flag is set, the inference rule UR-resolution (unitresulting resolution) (along with any other inference rules that are set) is used to generate new clauses.

para_into - default clear. If this flag is set, the inference rule "paramodulation into the given clause" (along with any other inference rules that are set) is used to generate new clauses. When using paramodulation, one should include the appropriate clause for reflexivity of equality, for example, $x=x$.

para_from - default clear. If this flag is set, the inference rule "paramodulation from the given clause" (along with any other inference rules that are set) is used to generate new clauses. When using paramodulation, one should include the appropriate clause for reflexivity of equality, for example, $\mathbf{x}=\mathbf{x}$.

demod_inf - default clear. If this flag is set, demodulation is applied, as if it were an inference rule, to the given clause. This is useful when term rewriting is the main objective. When this flag is set, the given clause is copied, then processed just like any newly generated clause. 


\subsubsection{Paramodulation Flags}

para_from_left - default set. If this flag is set, paramodulation is allowed from the left sides of equality literals. (Applies to both para_into and para_from inference rules.)

para_from_right - default set. If this flag is set, paramodulation is allowed from the right sides of equality literals. (Applies to both para_into and para_from inference rules.)

para_into_left - default set. If this flag is set, paramodulation is allowed into left sides of positive and negative equalities. (Applies to both para_into and para_from inference rules.)

para_into_right - default set. If this flag is set, paramodulation is allowed into right sides of positive and negative equalities. (Applies to both para_into and para_from inference rules.)

para_from_vars - default clear. If this flag is set, paramodulation from variables is allowed. Warning: setting this option may produce too many paramodulants. (Applies to both para_into and para_from inference rules.)

para_into_vars - default clear. If this flag is set, paramodulationinto variables is allowed. Warning: setting this option may produce too many paramodulants. (Applies to both para_into and para_from inference rules.)

para_from_units_only - default clear. If this flag is set, paramodulation is allowed only if the from clause is a unit (equality). (Applies to both para_into and para_from inference rules.)

para_into_units_only - default clear. If this flag is set, paramodulation is allowed only if the into clause is a unit. (Applies to both para_into and para_from inference rules.)

para_skip_skolem - default clear. If this flag is set, paramodulation is never allowed into subterms of Skolem expressions [16]. (Applies to both para_into and para_from inference rules.)

para_ones_rule - default clear. If this flag is set, paramodulation obeys the 1's rule. (The 1's rule is a special-purpose strategy for problems in combinatory logic; its usefulness has not been demonstrated elsewhere.) (Applies to both para_into and para_from inference rules.)

para_all - default clear. If this flag is set, all occurrences of the into term are replaced with the replacement term. (Applies to both para_into and para_from inference rules.)

\subsubsection{Flags for Handling Generated Clauses}

(Sec. 6.1.5 describes equality-related flags for handling generated clauses.)

detailed_history - default set. This flag affects the parent lists in clauses that 
are derived by binary_res, para_from, or para_into. If the flag is set, the positions of the unified literals or terms are given along with the IDs of the parents. See Sec. 15 for examples.

order_history - default clear. This flag affects the order of parent lists in clauses that are derived by hyperresolution, negative hyperresolution, or UR-resolution. If the flag is set, then the nucleus is listed first, and the satellites are listed in the order in which the corresponding literals appear in the nucleus. If the flag is clear (or if the clause was derived by some other inference rule), the given clause is listed first.

unit_deletion - default clear. If this flag is set, unit deletion is applied to newly generated clauses. Unit deletion removes a literal from a newly generated clause if the literal is the negation of an instance of a unit clause that occurs in usable or sos. For example, the second literal of $p(a, x) \mid q(a, x)$ is removed by the unit $-q(u, v)$; but it is uot removed by the unit $-q(u, b)$, because that unification causes the instantiation of $x$. All such literals are removed from the newly generated clause, even if the result is the empty clause. (Unit deletion is not useful if all generated clauses are units.)

delete_identical_nested_skolem - default clear. If this flag is set. clauses with the nested Skolem property are deleted. A clause has the nested Skol.m property if it contains a a Skolem expression that (properly) contains an occurrence of its leading Skolem symbol. For example, if $f$ is a Skolem function, a clause containing a term $f(f(x))$ or a term $f(g(f(x))$ ) is deleted.

sort_literals - default clear. If this flag is set, literals of newly generated clauses are sorted-negative literals, then positive literals, then answer literals. The main purpose of this flag is to make clauses more readable. In some cases, this flag can speed up subsumption on non-unit clauses.

for_sub - default sct. If this flag is set, forward subsumption is applied during the processing of newly generated clauses. (Delete the new clause if it is subsumed by any clause in usable or sos.)

back_sub - default set. If this flag is set, back subsumption is applied during the processing of newly kept clauses. (Delete all clauses in usable or sos that are subsumed by the newly kept clause.)

factor - default clear. If this flag is set, factoring is applied in two ways. First, factoring is applied as a simplification rule to newly generated clauses. If a generated clause $C^{\prime}$ has factors that subsume $C$, it is replaced with its smallest subsuming factor. Second, it is applied as an inference rule to newly kept clauses. Note that unlike other inference rules, factoring is not applied to the given clause; it is applied to a new clause as soon as it is kept. All factors are generated in an iterative manner. Factoring is attempted on answer literals. If factor is set, a clause with $n$ literals will not cause a clause with fewer than $n$ literals to be deleted by subsumption.

\subsubsection{Demodulation and Ordering Flags}

demod_history - default set. If this flag is set, then when a clause is demodulated, 
the ID numbers of the demodulators are included in the derivation history of the clause.

order_eq - default clear. If this flag is set, equalities are flipped if the right side is heavier than the left. See Secs. 8.1.2 and 8.2.2 for the meaning of "heavier".

eq_units_both_ways - default clear. If this flag is set, unit equality clauses (both positive and negative) are sometimes stored in both orientations; the action taken depends on the flag order_eq. If order_eq is clear, then whenever a unit, say $\alpha=\beta$, is processed, $\beta=\alpha$ is automatically generated and processed. If order_eq is set, then the reversed equality is generated only if the equality cannot be oriented (see Secs. 8.1.2 and 8.2.2).

demod_linear - default clear. If this flag is set, demodulation indexing is disabled, and a linear searchs of demodulators are used when rewriting terms. With indexing disabled, if more than one demodulator can be applied to rewrite a term, then the one whose clause number is lowest is applied; this flag is useful when demodulation is used to do "procedural" things. With indexing enabled (the default), demodulation is much faster, but the order in which demodulators is applied is not under the control of the user.

demod_out_in - default clear. If this flag is set, terms are demodulated outsidein, left-to-right. In other words, the program attempts to rewrite a term before rewriting (left-to-right) its subterins. The algorithm is "repeat \{rewrite the leftmost outer-most rewritable teim $\}$ until no more rewriting can be done or the limit is reached". (The effect is like a standard reduction in lambda-calculus or in combinatory logic.) If this flag is clear, terms are demodulated inside-out (all subterms are fully demodulated before attempting to rewrite a term). (The evaluable conditional term \$IF (condition, then-value, clse-value) is an exception when inside-out demodulation is in effect. See Sec. 9.)

dynamic_demod - default clear. If this flag is set, some newly kept equalities are made into demodulators (Secs. 8.1.3 and 8.2.3). Setting this flag automatically sets the flag order_eq.

dynamic_demod_all - default clear. If this flag is set, OTTER attempts to make all newly kept equalities into demodulators (Sec. 8.1.3). Setting this flag automatically sets the flags dynamic_demod ald order_eq.

dynamic_demod_lex_dep - default clear. If this flag is set, dynamic demodulators may be lex-dependent or LrPo-dependent. See Secs. 8.1.3 and 8.2.3.

back_demod - default clcar. If this flag is set, back demodulation is applied to demodulators, usable, and sos whenever a new demodulator is added. Back demodulation is delayed until the inference rules are finished generating clauses from the current given clause (delayed until post_process). Setting the back_demod flag automatically sets the flags order_eq and dynamic_demod.

knuth_bendix - default clear. If this flag is set, OTTER's search will behave like a Knuth-Bendix completion procedure. This flag is really a metaflag; its only effect is to alter other flags as follows: set(para_from), set(para_into), set (para_from_left), clear (para_from_right), sot (para_into_left), 
clear (para_into_right), set (para_from_vars), set (eq_units_both_ways), set(dynamic_demod_all), set (back_demod), set (process_input), and set (1rpo). See Sec. 8.3 for more details.

Irpo - default clear. If this flag is set, then the lexicographic recursive path ordering (also called RPO with status) is used to compare terms. If this flag is clear, weight templates and lexicographic order are used (Secs. 8.2 and 8.3).

lex_order_vars - default clear. This flag affects lex-dependent demodulation and the evaluable functions and predicates that perform lexical comparisons. If this flag is set, then lexical ordering is a total order on terms; variables are lowest in the term order, with $x \prec y \prec z \prec u \prec v \prec w \prec v 6 \prec v 7 \prec v 8 \prec \cdots$. If this flag is clear, then a variable is comparabl only to another occurrence of the same variable; it is not comparable to other variables or to nonvariables. For example, $\$ \operatorname{LLT}(f(x), f(y))$ evaluates to $\$ T$ if and only if lex_order_vars is set. If lrpo is set, lex_order_vars has no effect on demodulation (Sec. 8.1.1).

symbol_elim - default clear. If this flag is set, then new demodulators are orjented, if possible, so that function symbols (excluding constants) are eliminated. A demodulator can eliminate all occurrences of a function symbol if the arguments on the left side are all different variables and if the function symbol of the left side does not occur in the right side. For example, the demodulators $g(x)=f(x, x)$ and $h(x, y)=f(x, f(y, f(g(x), g(y))))$ eliminate all occurrences of $g$ and $h$, respectively.

\subsubsection{Input}

check_arity - default set. If this flag is set, a warning is given if symbols have variable arities (different numbers of arguments in different places in the input). For example, the term $f(a, a(b))$ would be flagged. (Constants have arity 0 .) If this flag is clear, then variable arities are permitted; in the preceding term, the two occurrences of a would be treated as different symbols.

prolog_style_variables - default clear. If this flag is set, a name with no arguments in a clause is a variable if and only if it starts with $A$ through $Z$ (upper case) or with ..

echo_included_files - default set. If this flag is set, input files included with the include(filename) command are echoed in the same way as ordinary input.

simplify_fol - default set. If this flag is set, then some propositional simplification is attempted when converting input first-order formulas into clauses. The simplification occurs after Skolemization, during the CNF translation. If simplification detects a refutation, it will always produce the empty clause $\$ F$, but OTTER will not recognize the proof (i.e., give the proof message and stop) unless the flag process_input is set.

process_input - default clear. If this flag is set, input usable and sos clauses (including clauses from formula input) are processed as if they had been generated by an inference rule. (See the procedure for processing newly inferred clause in Sec. 
2.) The exceptions are (1) the following clause-processing options are not applied to input clauses: max_literals, max_weight, delete_identical_nested_skolem, and max_distinct_vars, (2) clauses input on list usable remain there if retained, and (3) some output appears even if the output flags (Sec. 6.1.7) are clear.

\subsubsection{Output Flags}

very_verbose - default clear. If this flag is set, a tremendous amount of information about the processing of generated clauses is output.

print_kept - default set. If this flag is set, new clauses are output if they are retained (if they pass all retention tests).

print_proofs - default set. If this flag is set, all proofs that are found are printed to the output file. If this flag is clear, no proofs are printed.

print_new_demod - default set. If this flag is set, demodulators that are adjoined during the search (dynamic_demod) are printed. New demodulators are always printed during input processing.

print_back_demod - default set. If this flag is set, clauses are printed as they are back demodulated. Back-demodulated clauses are always printed during input processing.

print_back_sub - default set. If this flag is set, clauses are printed if they are back subsumed. Back-subsumed clauses are always printed during input processing.

display_terms - default clear. If this flag is set, all clauses and terms are printed in pure prefix form (Sec. 4.3). This feature can be useful for debugging the input.

pretty_print - default clear. If this flag is set, clauses are output in an indented form that is sometimes easier to read. The parameter pretty_print_indent (default 4) specifies the number of spaces for each indent level.

bird_print - default clear. If this flag is set, terms constructed with the binary function a are output in combinatory logic notation (without the function symbol $a$, and left associated unless otherwise indicated). For example, the clause $a(a(a(S, x), y), z)=a(a(x, z), a(y, z))$ is output as $S x y z=x z(y z)$.

Terms cannot be input in combinatory logic notation.

\subsubsection{Indexing Flags}

index_for_back_demod - default set. If this flag is set, all nonvariable terms in all clauses are indexed so that the appropriate ones can be quickly retrieved when applying a dynamic demodulator to the clause space (back demodulation). This type of indexing can use a lot of memory. If the flag is clear, back demodulation still works, but it is much slower.

for_sub_fpa - default clear. If this flag is set, FPA indexing is used for forward subsumption. If this flag is clear, discrimination tree indexing is used. Setting this flag can decrease the amount of memory required by OTTER. Discrimination 
tree indexing can require a lot of memory, but it is usually much faster than FPA indexing.

no_fapl -- default clear. If this flag is set, positive literals are not indexed for unit conflict or back subsumption. This option should be used only when no negative units will be generated (as with hyperresolution), back subsumption is disabled, and discrimination tree indexing is being used for forward subsumption. This option can save a lit tle time and memory.

no_fanl - default clear. If this flag is set, negative literals are not indexed for unit conflict or back subsumption. This option should be used only when no positive units will be generated (as with negative hyperresolution), back subsumption is disabled, and discrimination tree indexing is being used for forward subsimption. This option can save a little time and memory.

\subsubsection{Miscellaneous Flags}

control_memory - default clear. If this flag is set, then the automatic memorycontrol feature is enabled (Sec. 16).

order_hyper - default set. If this flag is set, then the inference rules hyper_res and neg_hyper_res are constrained by an ordering strategy. A literal in a satellite is allowed to resolve only if it is maximal in the satellite. (A literal is maximal in a clause if and only if there is no larger literal.) The ordering uses only the lexical value (as in the lex command or the default, Sec. 5.5) of the predicate symbol. (This flag is irrelevant for positive hyperresolution with a Horn set.)

propositional - default clear. If this flag is set, OTTER assumes that all clauses are propositional, and it makes some optimizations. The user should set this flag only when all clauses are propositional; otheruise OTTER may make unsound inferences and/or crash.

really_delete_clauses - default clear. If this flag is clear, clauses that are deleted by back subsumption or back demodulation are not really removed from memory; they are retained in a special place so that they can be printed if they occur in a proof. If the job involves much back subsumption or back demodulation and if memory conservation is important, these "deleted" clauses can be removed from memory by setting this flag (and any proof containing such a clause will not be printed in full).

atom_wt_max_args - default clear. If this flag is set, the default weight of an atom (the weight if no template matches the atom) is 1 plus the maximum of the weights of the arguments. If this flag is clear, the default weight of an atom is 1 plus the sum of the weights of the arguments.

term_wt_max_args -- default clear. If this flag is set, the default weight of a term (the weight if no template matches the atom) is 1 plus the maximum of the weights of the arguments. If this flag is clear, the default weight of a term is 1 plus the sum of the weights of the arguments.

free_all_mem - default clear. If this flag is set, then at the end of the search, 
most dynamically allocated memory is returned to the memory managers. This flag is used mainly for debugging, in particular, to help find memory leaks. Setting this flag will not cause OTTER to use less memory.

\subsection{Parameters}

Parameters are integer-valued options. In the descriptions that follow, $n$ is the value of the parameter, and MAX_INT is a large integer, usually the size of the largest normal integer on the user's computer.

\subsubsection{Monitoring Progress}

report - default -1 , range $[-1 . . \mathrm{MAX}\lrcorner \mathrm{NT}]$. If $n>0$, then statistics are output approximatcly every $n$ CPU seconds. The time is not exact, because statistics will be output only after the current given clause is finished. This feature can be used in conjunction with UNIX programs such as grep and awk to conveniently monitor OTTER jobs.

\subsubsection{Placing Limits on the Search}

max_seconds - default -1 , range $[-1 . . \mathrm{MAX}\lrcorner \mathrm{NT}]$. If $n \neq-1$, the search is terminated after about $n$ CPU seconds. The time is not exact, because OTTER will wait until the current given clause is finished before stopping.

max_gen - default -1 , range [-1..MAX_JNT]. If $n \neq-1$, the search is terminated after about $n$ clauses lave been generated. The number is not exact, because OTTER will wait until it is finished with the current given clause before stopping.

max_kept - default -1 , range [-1..MAX $\lrcorner \mathrm{NT}]$. If $n \neq-1$, the search is terminated after about $n$ clauses have been kept. The number is not exact, because OTTER will wait until it is finished with the current given clause before stopping.

max_given - default -1 , range $[-1 . . \mathrm{MAX}\lrcorner \mathrm{NT}]$. If $n \neq-1$, the search is terminated after $n$ given clauses have been used.

max_mem - default -1 , range [-1..MAX」NT]. If $n \neq-1$, OTTER will terminate the search before more than $n$ kilobytes have been dynamically allocated (mal1oc).

\subsubsection{Limits on Properties of Generated Clauses}

max_literals - default -1 , range $[-1 \ldots \mathrm{MAX}\lrcorner \mathrm{NT}]$. If $n \neq-1$, new clauses are discarded if they contain more than $n$ literals.

max_weight - default MAX_INT, range [-MAX_INT..MAX_INT]. New clauses are discarded if their weight is more than $n$. The weight list purge_gen or the weight list pick_and_purge is used to weigh clauses (both lists may not be present; see Sec. 10). 
max_distinct_vars - default -1 , range $[-1$...MAX」NT]. If $n \neq-1$, new clauses are discarded if they contain more than $n$ distinct variables.

\subsubsection{Indexing Parameters}

fpa_literals - default 8 , range [0..100]. $n$ is the FPA indexing depth for literals. (FPA literal indexing is used for resolution inference rules, back subsumption, and unit conflict. It is also used for forward subsumption if the flag for_sub_fpa is set.) If $n=0$, indexing is by predicate symbol only; if $n=1$, indexing looks at the predicate symbol and the leading symbols of the arguments of the literal, and so on. Greater indexing depth requires more memory, but it can be faster. Changing this parameter will not change the clauses that are generated or kept.

fpa_terms - default 8 , range [0..100]. $n$ is the FPA indexing depth for terms. (FPA term indexing is used for paramodulation inference rules and back demodulation.) If $n=0$, indexing is by function symbol only; if $n=1$, indexing looks at the function symbol and the leading symbols of the arguments of the term, and so on. Greater indexing depth requires more memory, but it can be faster. Changing this parameter will not change the clauses that are generated or kept.

\subsubsection{Miscellaneous Parameters}

pick_given_ratio - default -1 , range $[-1 \ldots \mathrm{MAX}\lrcorner \mathrm{NT}]$. This parameter causes some given clauses to be selected by weight and others in a breadth-first manner. If $n \neq-1, n$ given clauses are are selected by (smallest pick_given) weight, then the first clause in sos is selected as given clause, then $n$ given clauses are selected by weight, etc. This method allows heavy clauses to enter into the search while focusing mainly on light clauses. It combines breadth-first search (flag sos_queue) and best-first search (default selection by weight). If $n$ is -1 , then the clause with smallest pick_given weight is always selected.

interrupt_given - default -1 , range [-1..MAX $\lrcorner \mathrm{NT}]$. If $n>0$, then after $n$ given clauses have been used, OTTER goes into its interactive mode (Sec. 14).

demod_limit - default 1000 , range $[-1 \ldots \mathrm{MAX}\lrcorner \mathrm{NT}]$. If $n \neq-1, n$ is the maximum number of rewrites that will be applied when demodulating a clause. The count includes $\$$ symbol evaluation. If $n$ is -1 , there is no limit. A warning message is printed if OTTER attempts to exceed the limit.

max_proofs - default 1 , range $[-1 \ldots \mathrm{MAX}\lrcorner \mathrm{NT}]$. If $n=1$, OTTER will stop if it finds a proof. If $n>1$, then OTTER will not stop when it has found the first proof; instead, it will try to keep searching until it has found $n$ proofs. (Some of the proofs may in fact be identical.) (Because forward subsumption occurs before unit conflict, a clause representing a truly different proof may be discarded by forward subsumption before unit conflict detects the proof.) If $n=-1$, OTTER will find as many proofs as it can (within other constraints).

min_bit_width-- default bits-per-long, range [0..bits-per-long]. When the evaluable bit operations (Sec. 9) produce a new bit string, leading zeros are suppressed under 
the constraint that $n$ is the minimum string length.

neg_weight - default 0 , range [-MAX_NT..MAX_JN] $n$ is the additional weight (positive or negative) that is given to negated literals. Weight templates cannot be used for this purpose, because the negation sign on a literal cannot occur in weight templates. (Atoms, not literals, are weighed with weight templates; see Sec. 10.)

pretty_print_indent - default 4 , range [0..16]. See flag pretty_print, Sec. 6.1.7.

stats_level - default 2, range [0..4]. This indicates the level of detail of statistics printed in reports and at the end of the search. If $n=0$, no statistics are output; if $n=1$, a few important search and time statistics are output; if $n=2$, all search and time statistics are output; if $n=3$, search, time, and memory statistics are output; and if $n=4$, search, time, and memory statistics and option values are output. This parameter does not affect the speed of OTTER, because all statistics are always kept.

\section{Demodulation}

Basic demodulation is straightforward, but there are many variations and enhancements whose descriptions are scattered throughout this manual. This section (which is mostly redundant) lists some overall comments on demodulation and points the reader to the appropriate sections on variations and enhancements.

The Equality Symbol. The binary symbol = (which can be used as an infix functor) and any name that starts with eq, EQ, or Eq, when used as a binary predicate symbol, is recognized as an equality predicate by demodulation.

When and How It Is Applied. Demodulation is applied, using equalities in the list demodulators, to every clause that is generated by an inference rule. Also, when the flag demod_inf (Sec. (6.1.2) is set, demodulation is, in effect, treated as an inference rule.

Demodulation of Atomic Formulas. Atomic formulas (literals with any negation sign removed) can be demodulated. Useful examples are

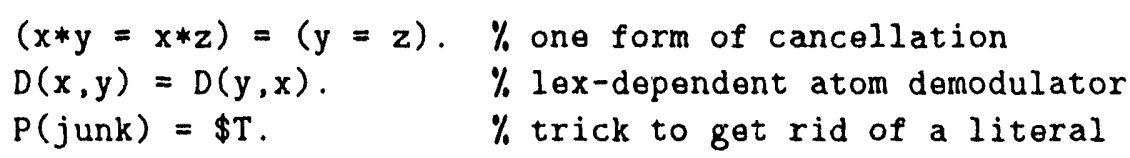

The appropriate clause simplification occurs if the right side of an atom demodulator is one of the Boolean constants $\$ T$ or $\$ F$. Negated literals cannot be demodulated, but the atom of a negative literal can be demodulated. 
Inside-out or Outside-in. The user has the option of having terms rewritten inside-out or outside-in. (See the description of the flag demod_out_in in Sec. 6.1.5.) Although the choice makes little difference for many applications, I nearly always recommend inside-out. Outside-in can be much faster in cases where the left side of the demodulator has a variable not in the right side.

Order of Demodulators. By default, demodulation uses an indexing mechanism to find demodulators that can rewrite a given term; if more than one demodulator can apply, the user has no control over which one is used. If the user wishes to order the set of demodulators for application, he or she can set the flag demod_linear (Sec. 6.1.5).

Dynamic Demodulation and Back Demodulation. Positive equality units derived during the search can be made into demodulators (Secs. 6.1.5, 8.1.3, and 8.2.3). Demodulators adjoined during the search can be used to rewrite previously derived clauses (Sec. 6.1.5).

Termination. With the default ad hoc ordering, demodulation is not guaranteed to terminate by itself. Therefore, a parameter (demod_limit) specifies the maximum number of rewrite steps that will be applied to a clause. With the lexicographic recursive path ordering (flag $1 \mathrm{rpo}$ ), demodulation will always terminate by itself. (Even with Irpo, the parameter demod_limit has effect, because demodulation sequences can have an unreasonable number of steps.)

Introduction of New Variables. A demodulator introduces new variables if it has variables on the right side that do not occur on the left. The LRPO does not allow demodulators to introduce new variables. The default ordering allows variable introductions only for input demodulators.

Lex- and LRPO-dependent Demodulation. Ordinary demodulators are used unconditionally; they usually simplify or canonicalize regardless of the context in which they are applied. But some equalities that are not normally thought of as rewrite rules can be used as such and are applied only if the application produces a "better" term. These are called lex-or LRPO-dependent demodulators (depending on whether the flag Irpo is set). For example, commutativity of an operation, say $x+y=y+x$, can be used to rewrite $b+a$ to $a+b$ if $a+b \prec b+a$. See Secs. 6.1.5, 8.1.4, and 8.2.4. Do not confuse this type of demodulation with conditional demodulation.

Demodulation of Evaluable Terms. OTTER has many built-in function and predicate symbols for doing arithmetic, logic operations, bit operations, and other operations. The evaluation of terms containing these built-in symbols is done as a part of demodulation (Sec. 9). 
Conditional Demodulation. Demodulators can be written with conditions as

condition $\rightarrow \alpha=\beta$.

The demodulator is applied only if the condition, instantiated with the matching substitution, demodulates to \$T (meaning true). This is a "fringe feature", and it has not been heavily used (Sec. 17.4).

Demodulation as Equational Programming. O'TTen's demodulation, especially with the evaluable symbols, can be used as a general-purpose (although not particularly efficient or convenient) equational programming system (Sec. 9). I have not seen cases where this is useful in the context of a traditional refutation search, but I have found it to be very useful for various symbolic programming tasks, particularly with hyperresolution.

Demodulation to Delete Clauses. Demodulation can be used as a trick to overcome one of the deficiencies of the weighting mechanism (Sec. 10) to discard undesired clauses. Weighting does not implement a true match (one-way unification) operation. If the user wishes to discard every clause that contains an instance of a particular term, say $f(x, x)$, a demodulator, say $f(x, x)=$ junk, can be input along with a weight template that gives junk a purge gen weight higher than max_woight. (When using this and similar tricks, the user must make sure that the clauses containing junk are really discarded by weighting or another means; on occasion we have found proofs that are incorrect because they depend on junk.)

\section{Ordering and Dynamic Demodulation}

This section contains a more complete explanation of the options lex_order_vars, order_eq, symbol_elim, dynamic_demod, dynamic_demod_all, lrpo, and dynamic_demod_lex_dep. It gives all the rules built in and optional for orienting equality literals and deciding which equalities will be dynamic demodulators. OrTer uses two kinds of term ordering.

ad hor ordering. This is a collection of ordering methods that we have acrumulated through many years of experimentation. The methods do not have a substantial theoretical foundation, but they are useful in many cases. 'This is the default ordering; it is presented in Sec. 8.1.

LRPO. 'This is the lexicographic recursive path ordering (also called RPO with status). It has nice theoretical properties and is easier to use than the ad hoc ordering, but it is more computationally expensive. The trRo ordering is enabled with the llag 1rpo; it is described in See. 8.2.

Both hinds of term ordering use an ordering on constant and function symbols. the lex command (Sec. 5.5) is used to assign an ordering on symbols. For example, the command 
$\operatorname{lex}([a, b, c, d, \operatorname{or}(\ldots)]$,$) .$

specifies $a \prec b \prec c \prec d \prec$ or (or is a binary function symbol). If a lex command is given, all constant and function symbols in terms that will be compared must be included. If a lex command is not given, OTTER uses the following default ordering.

[constants, high-arity, $\cdots$, binary, unary]

Within arity, the lexicographic Ascul ordering (i.e., the C library routine strcomp()) is used.

The methods for orienting equalities and for determining dynamic and lexdependent demodulators apply to all inferred clauses; if the flag process_input is set, they also apply to input usablo and sos clauses.

In this section, $\alpha$ and $\beta$ always refer to the left and right arguments, respectively, of the equality literal under consideration; $w t(\gamma)$ refers to the weight of $\gamma$ using weight_list_terms; vars $(\gamma)$ is the set of variables in $\gamma$. The symbols $\succ$ and $\prec$ are used for several orderings; the one referred to should be clear from the context.

Table 6 is a quick reference guide to the ordering mechanisms presented in Secs. 8.1 and 8.2 .

Table 6: Quick Reference to Ordering

\begin{tabular}{|l|l||l||l|}
\hline \multicolumn{2}{|c||}{ Situation } & \multicolumn{1}{c|}{ Ad Hoc } & \multicolumn{1}{|c|}{ LRPO } \\
\hline \hline Input demods & flip? & no & if $\alpha<\beta$ \\
\cline { 2 - 4 } & lex-dependent? & if ident-x-vars & if neither is greater \\
\hline \multirow{4}{*}{ Orienting eqs (order_eq set) } & $\begin{array}{l}\text { Hip if sym-elim, } \\
\text { occurs-in, or wt-lex-ord }\end{array}$ & flip if $\alpha \prec \beta$ \\
\hline \multirow{4}{*}{ Dynamic demod? } & d_d_all clear & $\begin{array}{l}\text { if oriented, var-subset, } \\
\text { and } w t(\beta) \leq 1\end{array}$ & if $\alpha \succ \beta$ \\
\cline { 2 - 4 } & d_d_all set & if oriented and var-subset & if $\alpha \succ \beta$ \\
\cline { 2 - 4 } & lex-dependent? & $\begin{array}{l}\text { if ident-x-vars and } \\
\text { dynamic_desnod_all set }\end{array}$ & $\begin{array}{l}\text { if neither is greater, } \\
\text { and var-subset }\end{array}$ \\
\hline Apply lex-dependent demod? & lex-order $(\alpha \sigma, \beta \sigma)$ & $\alpha \sigma \succ \beta \sigma$ \\
\hline Lex \$ evaluation & lex-order & lex-order \\
\hline
\end{tabular}

\subsection{Ad Hoc Ordering}

\subsubsection{Term Ordering (Ad Hoc)}

Two types of ad hoc term ordering are used: lex-order and weight-lex-order. The user does not have a choice between these two; the one that is applied depends on the context, as described in the following subsections.

lex-order. This is a basic lexicographic extension of the symbol order. To compare two terms, read them left to right, and stop at the first symbols where they differ; the relationship of those symbols determines the term order. The treatment of variables depends on the flag lex-order-vars: 
lex_order_vars is set. Variables are the lowest in the symbol ordering, with $x \prec y \prec z \prec u \prec v \prec w \prec v 6 \prec v 7 \prec v 8 \prec \cdots$. Since the order on symbols is total (any two symbols are comparable), the lexical order on terms is total (any two terms are comparable). Note that applying a substitution to a pair of terms may change their relative order.

lex_order_vars is clear (the default). A variable is comparable only to itself and to a term that contains the variable. The order on terms is partial. Note that if $t_{1} \prec t_{2}$, and if $\sigma$ is any substitution, then $t_{1} \sigma \prec t_{2} \sigma$.

weight-lex-order. In comparing two terms, they are first weighed with weight_list_terms. If one term is heavier, it is greater in the order. If the terms have equal weight, they are compared with respect to the lex-order as if lex-order-vars is clear.

\subsubsection{Orienting Equalities (Ad Hoc)}

If the flag order_eq is set and Irpo is clear, then equality literals (both positive and negative) in inferred clauses are processed as follows.

1. If the symbol_el im flag is set and if the equality is a symbol-eliminating type (Sec. 6.1.5), the equality is oriented in the appropriate direction.

2. If one argument is a proper subterm of the other argument, the equality is oriented so that the subterm is the right-hand argument.

3. If one argument is greater in the weight-lex-order, say $\gamma \succ \delta$, the equality is oriented with $\gamma$ as the left side.

The preceding steps do not apply to equalities input on the list demodulators.

\subsubsection{Determining Dynamic Demodulators (Ad Hoc)}

A dynamic demodulator is a demodulator that is inferred rather than input. If either of the flags dynamic_demod or dynamic_demod_all is set, the flag order_eq will also be set, and OTTEn will attempt to make some or all inferred positive equality units into demodulators. If the flag process_input is set, the procedure applies to input usable and sos equalities. The procedure assumes that equalities have already been oriented.

1. If the flag symbol_elim is set and if $\alpha=\beta$ is symbol-eliminating, the equality becomes a demodulator.

2. If $\beta$ is a proper subterm of $\alpha$, the equality becomes a demodulator.

3. If $\alpha \succ \beta$ in the weight-lex-order, and if $\operatorname{vars}(\alpha) \supseteq \operatorname{vars}(\beta)$,

(a) if dynamic_demod_all is set, the equality becomes a demodulator; 
(b) if dynamic_demod_all is clear and if $w t(\beta) \leq 1$, the equality becomes a demodulator.

4. If dynamic_demod_lex_dep and dynamic_demod_all are both set, if $\alpha$ and $\beta$ are identical-except-variables (Sec. 8.1.4), and if $\operatorname{vars}(\alpha) \supseteq \operatorname{vars}(\beta)$, the equality becomes a lex-dependent demodulator.

\subsubsection{Lex-dependent Demodulation (Ad Hoc)}

Two terms are identical-except-variables if they are identical after replacing all occurrences of variables with $x$. An input or dynamic demodulator is lex-dependent only if $\alpha$ and $\beta$ are identical-except-variables. (See Sec. 8.1.3 for determining lexdependent dynamic demodulators.) A lex-dependent demodulator applies to a term only if the replacement term is smaller in the lex-order. In particular, OTTER will apply a lex-dependent demodulator $\alpha=\beta$ if and only if $\alpha \sigma \succ \beta \sigma$ in the lex-order, where $\sigma$ is the matching substitution.

For example, in the presence of the lex command and the (lex-dependent) demodulators

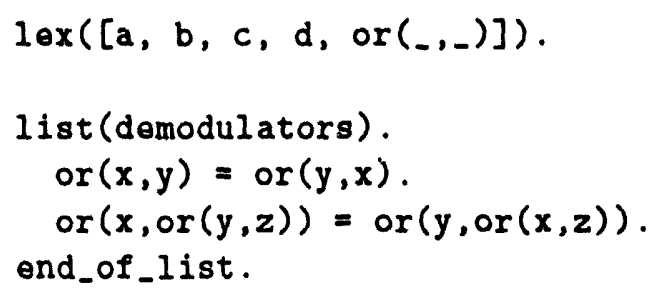

the term or $(\operatorname{or}(d, b), \operatorname{or}(a, c))$ will be demodulated to or $(a, \operatorname{or}(b, \operatorname{or}(c, d)))($ in several steps).

\subsection{LRPO}

\subsubsection{Term Ordering (LRPO)}

The lexicographic recursive path ordering (LRPO, or RPO with status) $[5,8,10]$ is a method for comparing terms. The important theoretical property of LRPO is that it is a termination ordering. That is, let $R$ be a set of demodulators in which in each demodulator, the left side is LRPO-greater than the right side; then demudulation (applying the demodulators left to right) is guaranteed to terminate.

To use LRPO one typically uses the lex command (Sec. 5.5) to assign an ordering on constant and function symbols. If the lex command is not present, OTTER assigns an ordering (which is frequently ineffective). (OTTER uses a total ordering on symbols that is fixed at input time. Other implementations of LRPO use partial orderings or dynamically changing orderings.)

With respect to LRPO, function symbols can have either left-to-right status (the default) or multiset status. The command 1rpo_multiset_status (symbol_list) gives 
symbols multiset status.

LRPo comparison is used when orienting equality literals, deciding whether an equality should be a demodulator or an LRPO-dependent demodulator, and deciding whether to apply an LRPO-dependent demodulator. LRPO comparison is never used when evaluating the functions/predicates that perform lexical comparison (\$LLT, \$LGT, etc.).

\subsubsection{Orienting Equalities (LRPO)}

If the flag order_eq is set and if one argument of the equality literal (positive or negative) is greater in the LRPO order, the greater argument is placed on the left side. This rule applies to input demodulators, to inferred clauses, and, if the flag process_input is set, to input usable and sos clauses.

\subsubsection{Determining Dynamic Demodulators (LRPO)}

If the flag dynamic_demod is set, OTTER attempts to make all equalities into demodulators (dynamic_demod_all is ignored when lrpo is set). If $\alpha \succ \beta$ in the LRPO order, the derived equality becomes a demodulator ( $\alpha$ is not LRPo-less-than $\beta$, because orienting has already occurred). If dynamic_demod_lex_dep is set, if neither argument is LRPO-less-than the other, and if every variable that occurs in $\beta$ also occurs in $\alpha$, the derived equality becomes an LRPO-dependent demodulator.

\subsubsection{LRPO-dependent Demodulation (LRPO)}

An LRPO-dependent demodulator is allowed to rewrite a term if and only if its application produces an LRPO-less-than term.

\subsection{Knuth-Bendix Completion}

The Kinuth-Bendix completion procedure [12] attempts to transform a set $E$ of equalities into a terminating, canonical set of rewrite rules (demodulators). If it is successful, the resulting set of rewrite rules, a complete set of reductions, is a decision procedure for equality of terms in the theory $E$. There are many variations and refinements of the Kinuth-Bendix procedure.

Setting the flag knuth_bendix causes OTTER to automatically alter a set of options so that its search will behave like a Knuth-Bendix completion procedure. If OTTER's search stops because its sos list is empty, and if certain other conditions are met, then the resulting set of equalities is a complete set of reductions. (OTTER was not designed to implement a completion procedure, and it has not been optimized for completion.)

Claim. If (1) the set $E$ of equalities, along with $\mathbf{x}=\mathbf{x}$, is input in list sos, (2) flag knuth_bendix is set, (3) other options that are changed from the defaults do not 
affect the search, (4) OTTER stops with "sos empty", and (5) other than $x=x$, the final usable list is the same as the final demodulators list, then the demodulators list is a complete set of reductions for $E$.

Here is an input file that causes OTTER to search for and quickly find a complete set of reductions for free groups. Note that the predeclared (right associative) infix operator $*$ is used.

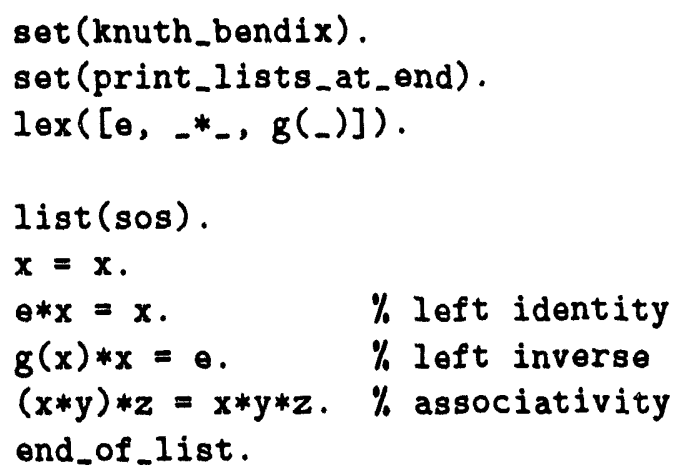

The critical issue in most applications of the Knuth-Bendix completion procedure is the choice of ordering scheme and/or the specific ordering on symbols. Note, in this case, that if the lex command is absent, the default symbol ordering suffices because it is essentially the same as the one specified.

The knuth-bendix flag is also very useful when trying to prove equational theorems. (Many open problems have been solved at Argonne in this way; see, e.g., [17]). When using knuth_bendix to search for proofs, we are not bound by the conditions listed in the above claim; in fact, we usually apply additional strategies such as limiting the size of retained equalities, being more selective about making equalities into demodulators, and disabling LRPO ordering.

With the following input file, OTTER uses the knuth-bendix option to prove the difficult half of a group theory theorem of Levi: The commutator operation is associative if and only if the commutator of any two elements lies in the center of the group. (A textbook proof can be found in [13].) Note that, contrary to common practice, the symbol order does not cause the definition of the commutator operation $h(\ldots$,$) to be used as a rewrite rule to eliminate commutator expressions in h$. Note also that weight templates are used to eliminate clauses containing terms with particular structures; this decision is purely heuristic, derived from experimentation and intuition. OTTER finds a proof in about half an hour on a SPARCstation 2 and uses about 6 megabytes of memory.

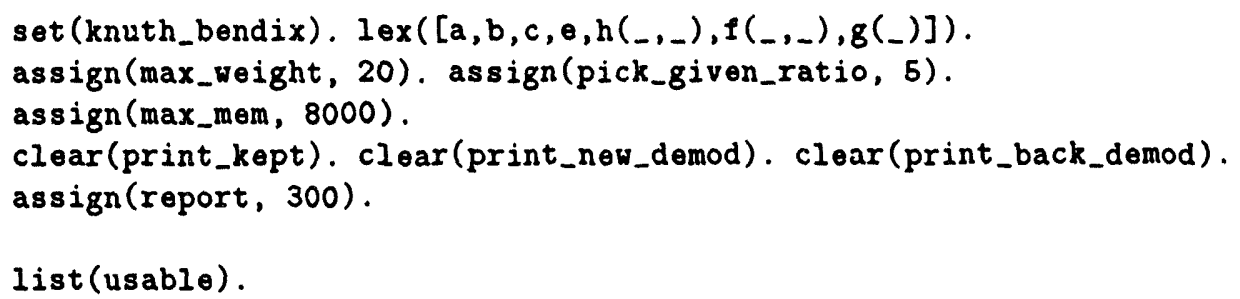




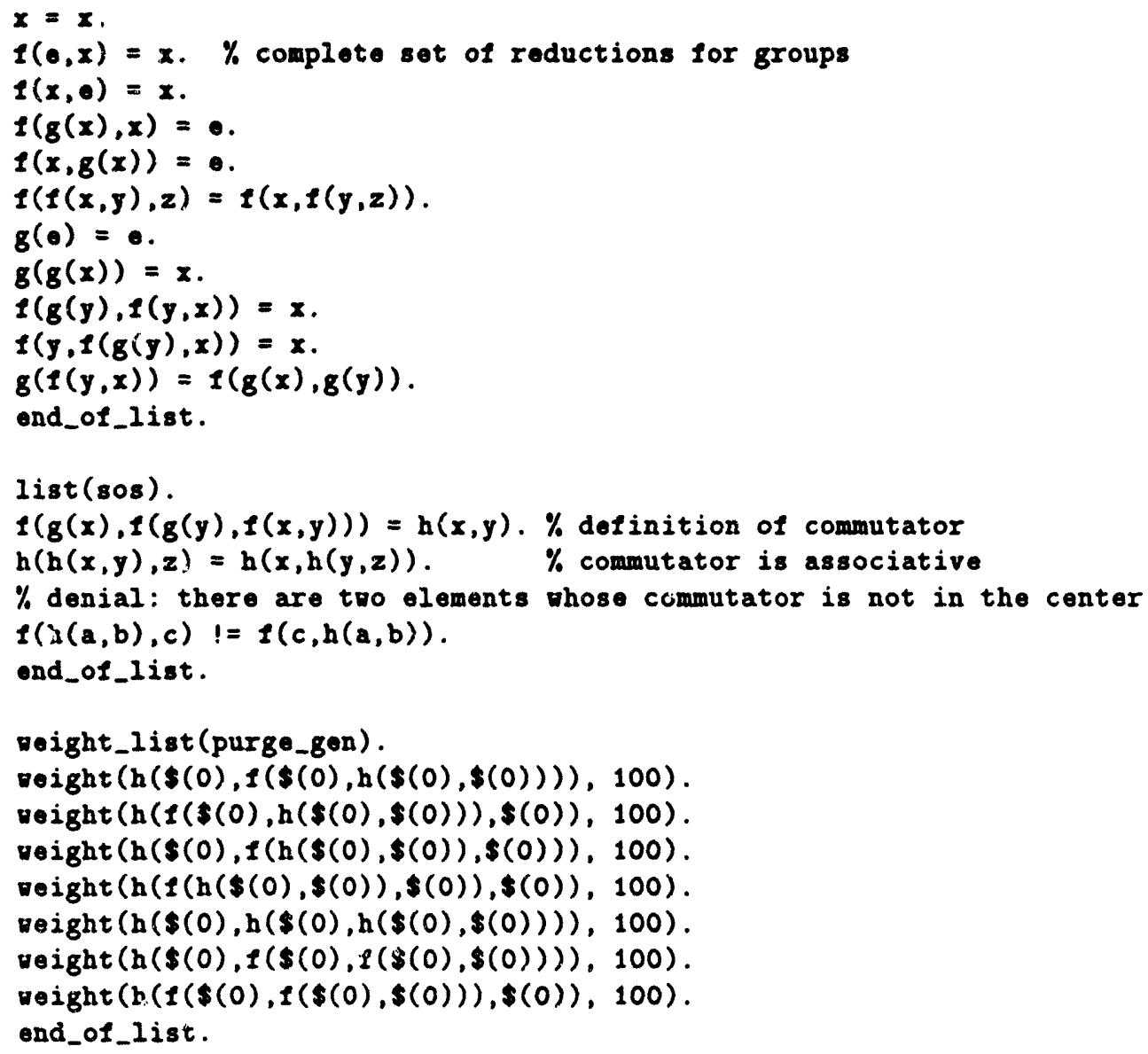

\section{Evaluable Functions and Predicates (\$SUM, \$LT, ..)}

OTTER can be used in a "programmed" mode that is quite different from normal refutational theorem proving. When using the programmed mode, one generally has in mind a particular method for solving a problem; and when writing clauses for the programmed mode, one generally knows exactly how they will be used by OTTER.

The programmed mode frequently involves a set of evaluable function and predicate symbols known as the $\$$-symbols (because each starts with \$). Examples are \$SUM and \$LT for integer arithmetic and \$AND for Boolean operations.

The evaluable symbols operate on four types of OTTER term: integer constants, bit-string constants, the Boolean constants $\$ T$ and $\$ F$, and arbitrary terms. The symbols that evaluate to type Boolean can occur either as function symbols or as predicate symbols. The integer and bit operations behave the same as the underlying C operations applied to the data type "long int" and "unsigned long int", respectively. Table 7 lists the evaluable functions and predicates by type.

Additional notes on the operations (unless otherwise stated, the term in question evaluates if all arguments demodulate/evaluate to the appropriate type): 
Table 7: Evaluable Functions and Predicates

\begin{tabular}{|l|l|}
\hline int $\times$ int $\rightarrow$ int & \$SUM, \$PROD, \$DIFF, \$DIV, \$MOD \\
\hline int $\times$ int $\rightarrow$ bool & \$EQ, \$NE, \$LT, \$LE, \$GT, \$GE \\
\hline bits $\times$ bits $\rightarrow$ bits & \$BIT_AND, \$BIT_OR, \$BIT_XOR \\
\hline bits $\times$ int $\rightarrow$ bits & \$SHIFT_LEFT, \$SHIFT_RIGHT \\
\hline bits $\rightarrow$ bits & \$BIT_NOT \\
\hline int $\rightarrow$ bits & \$INT_TO_BITS \\
\hline bits $\rightarrow$ int & \$BITS_TO_INT \\
\hline term $\times$ term $\rightarrow$ bool (lexical) & \$ID, \$LNE, \$LLT, \$LLE, \$LGT, \$LGE \\
\hline$\rightarrow$ bool & \$T, \$F \\
\hline bool $\times$ bool $\rightarrow$ bool & \$AND, \$OR \\
\hline bool $\rightarrow$ bool & \$TRUE, \$NOT \\
\hline term $\rightarrow$ bool & \$ATOMIC, \$INT, \$BITS, \$VAR, \$GROUND \\
\hline$\rightarrow$ int & \$NEXT_CL_NUM \\
\hline bool $\times$ term $\times$ term $\rightarrow$ term & \$IF \\
\hline
\end{tabular}

- int $\times$ int $\rightarrow$ int. The symbol \$SUM is addition, \$PROD is multiplication, \$DIFF is subtraction, \$DIV is integer division, and \$MOD is remainder.

- int $\times$ int $\rightarrow$ bool. These are the ordinary relational operations on integers. The symbol $\$ E Q$ is $=, \$ N E$ is $\neq$, , $\mathrm{LT}$ is $<, \$ \mathrm{LE}$ is $\leq, \$ \mathrm{GT}$ is $>$, and $\$ \mathrm{GE}$ is $\geq$.

- bits $\times$ int $\rightarrow$ bits. The shift operations \$SHIFT_LEFT and \$SHIFT_RIGHT shift the first argument by the number of places given by the second argument.

- bits $\times$ bits $\rightarrow$ bits. The symbols \$BIT_AND, \$BIT_OR, and \$BIT_XOR are the bitwise conjunction, disjunction, and exclusive-or operations.

- bits $\rightarrow$ bits. The symbol \$BIT_NOT is the one's complement operation on bit strings.

- int $\rightarrow$ bits. The symbol \$INTS_TO_BITS translates a decimal integer to a bit string.

- bits $\rightarrow$ int. The symbol \$BITS_TO_INT translates a hit string to the corresponding decimal integer.

- term $\times$ term $\rightarrow$ bool. The term always evaluates. These operations are analogous to the six operations in int $\times$ int $\rightarrow$ bool, except that the comparisons are lexical instead of arithmetic. The symbol $\$$ ID tests identity of terms. The lexical comparison is the same as in lex-dependent demodulation; in particular, the flag lex_order_vars (Secs. 6.1.5 and 8.1.1) has effect.

- $\rightarrow$ bool. The symbols $\$ \mathrm{~T}$ and $\$ \mathrm{~F}$ represent true and false. When they appear as literals or atomic formulas in clauses, the clauses are simplified as appropriate.

- bool $\rightarrow$ bool. The symbol \$TRUE is essentially a "no operation" on Boolean constants. It is used to trick hyperresolution into evaluating literals (see below). 
- term $\rightarrow$ bool. A term is \$ATOMIC iff it is a constant (including integer and bit string), a term is a \$INT iff it is an integer, a term is a \$BITS iff it is a string of $\{0,1\}$, a term is a $\$$ VAR iff it is a (unbound) variable, and a term is a \$GROUND iff it does not contain any variables.

- $\rightarrow$ int. The term \$NEXT_CL_NUM (no arguments) evaluates to the next integer that will be assigned as a clause identifier (this is useful for placing the ID of a clause within the clause).

- bool $\times$ term $\times$ term $\rightarrow$ term. The $\$ I F$ function is the if-then-else operator. When inside-out (the default) demodulation encounters a term $\$$ IF (condition, $\left.t_{1}, t_{2}\right)$, demodulation takes a path different from its normal inside-out behavior. The term condition is demodulated (evaluated); if the result is $\$ \mathrm{~T}$, the value of the $\$ I F$ term is the result of demodulating $t_{1}$; if the result is $\$ F$, the value of the $\$ I F$ term is the result of demodulating $t_{2}$; if the result is neither $\$ T$ nor $\$ F$, demodulation returns to its normal behavior. Note that if the condition evaluates to a Boolean value, demodulation deviates from its inside-out behavior, because just one of $t_{1}$ and $t_{2}$ is demodulated. (If demodulation were always outside-in, \$IF would not need to be built in, because it could be efficiently defined with the two demodulators if $(\$ T, x, y)=x$ and if $(\$ F, x, y)=y$.)

Evaluation occurs as part of the demodulation process. In particular, if demodulation comes across an evaluable term, say $\$ \operatorname{SUM}(2,3)$, it tries to convert the arguments into the appropriate type (integers for \$SUM); then if the arguments have the correct type, it rewrites the term to the result of the operation, in this case, just as if the demodulator $\$ \operatorname{SUM}(2,3)=5$ had been present. The evaluation mechanisms, along with ordinary demodulation, form a reasonably complete (although not particularly speedy or convenient) equational programming subsystem.

Evaluation/demodulation can also occur, in a very particular way, during hyperresolution. (Recall that lyperresolution takes a clause, the nucleus, with some negative literals, the conditions, and resolves each negative literal with a positive clause, producing a clause with no negative literals.) Just as evaluation during demodulation can be thought of as rewriting with an implicit demodulator, evaluation during hyperresolution can be thought of resolving with the implicit positive unit clause \$T (meaning "true"). The mechanism is this: if hyperresolution encounters a negative literal that has an evaluable predicate symbol, then it demodulates the atom (the literal without the sign); if the result of the demodulation is $\$ T$, then the literal is considered to have been resolved.

During hyperresolution, demodulation/evaluation is triggered by the presence of an evaluable literal. In many cases, however, the user defines a Boolean function that he or she wishes to trigger the mechanism. Consider the following definition of list membership, written as demodulators:

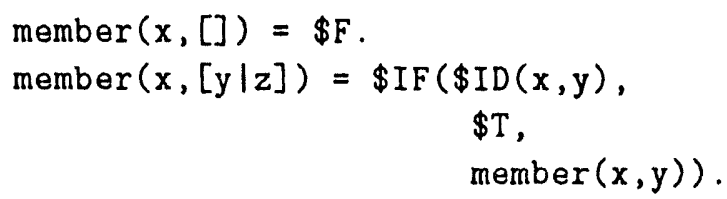


Because the symbol member is not evaluable, the demodulation/evaluation mechanism will not be activated; however, the unary evaluable predicate \$TRUE can be used in the following way to trigger demodulation/evaluation.

$$
-L_{1}|\cdots|-\$ \operatorname{TRUE} \text { (member (element, list)) }|\cdots|-L_{n} \mid M \text {. }
$$

Evaluable functions and predicates are useful to implement forward-chaining rulebased systems, for example, state-space search problems (Sec. 9.2).

Hyperresolution operates on the conditions (negative literals) in order, left to right. (The preceding sentence is not quite true, because the first step is typically resolution of a positive given clause with any one of the conditions, but for this paragraph, we may assume that it is true.) If a literal resolves or evaluates, the next literal is considered. If nothing more can be done with a literal, then hyperresolution backtracks to the preceding literal in search of an alternative. When a nucleus contains evaluable conditions, the order of the conditions is important both for efficiency and for actually deriving hyperresolvents. Evaluable conditions typically have variables that must be instantiated when nonevaluable literals are resolved. If an evaluable literal is too far to the left, its variables will not be sufficiently instantiated when hyperresolution encounters it, evaluation will fail, and possible paths to hyperresolvents will be blocked. If an evaluable literal is too far to the right, then hyperresolution can explore many paths that are sure to fail.

Technical Note and Advice. The evaluable symbols are an add-on feature rather than an integral part of OTTER. In particular, the objects that are manipulated (integers, bit strings, etc.) in most cases are stored by OTTER as character strings rather than as the appropriate data type. To evaluate a term, say $\$ \operatorname{SUM}(2,3)$, OTTER must find the strings " 2 " and " 3 " in a hash table, translate them to integers, add them, translate the result to the string "5", then look up "5", and possibly insert it into the hash table. This procedure is obviously much slower than it needs to be. If the user has a problem that requires a hundred million evaluations, he or she should consider using something else, including writing a special-purpose $\mathrm{C}$ program.

Warning. The evaluable symbols should not be thought of as theories "built in" to OTTER. As theories, they are very incomplete, and OTTER uses them only in very constrained ways.

\subsection{Using More Natural Expressions for Evaluation}

Writing complex evaluable expressions with $\$$-symbols can be quite tedious. Therefore, a feature was added that allows more natural expressions. The command make_evaluable copies the evaluation properties from a \$symbol to any other symbol of the same arity. The form of the command is

make_evaluable(any-symbol, evaluable-symbol).

The symbols in the command are given dummy arguments to specify the arity. The following list contains typical examples for integer arithmetic (assuming the symbols on the left are already known to be infix). 


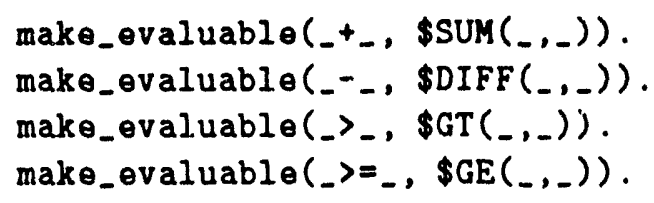

Warning 1. If a binary symbol that is recognized by paramodulation or demodulation as an equality symbol is given evaluation properties, it will no longer be recognized by paramodulation or demodulation. For example, if the command make_evaluable $\left(_{-}=_{-}, \$ E Q\left(_{-}, \ldots\right)\right.$ ) is issued, paramodulation and demodulation will not recognize $a=b$ as an equality. The convention is to use $==$ for evaluation.

Warning 2. This is not an "alias" mechanism; the symbols remain distinct for unification, matching, and identity testing.

\subsection{Evaluation Examples}

Equational Programming. The evaluable functions and predicates enable the use of equalities with demodulation as a general-purpose equational programming language. Here are some examples.

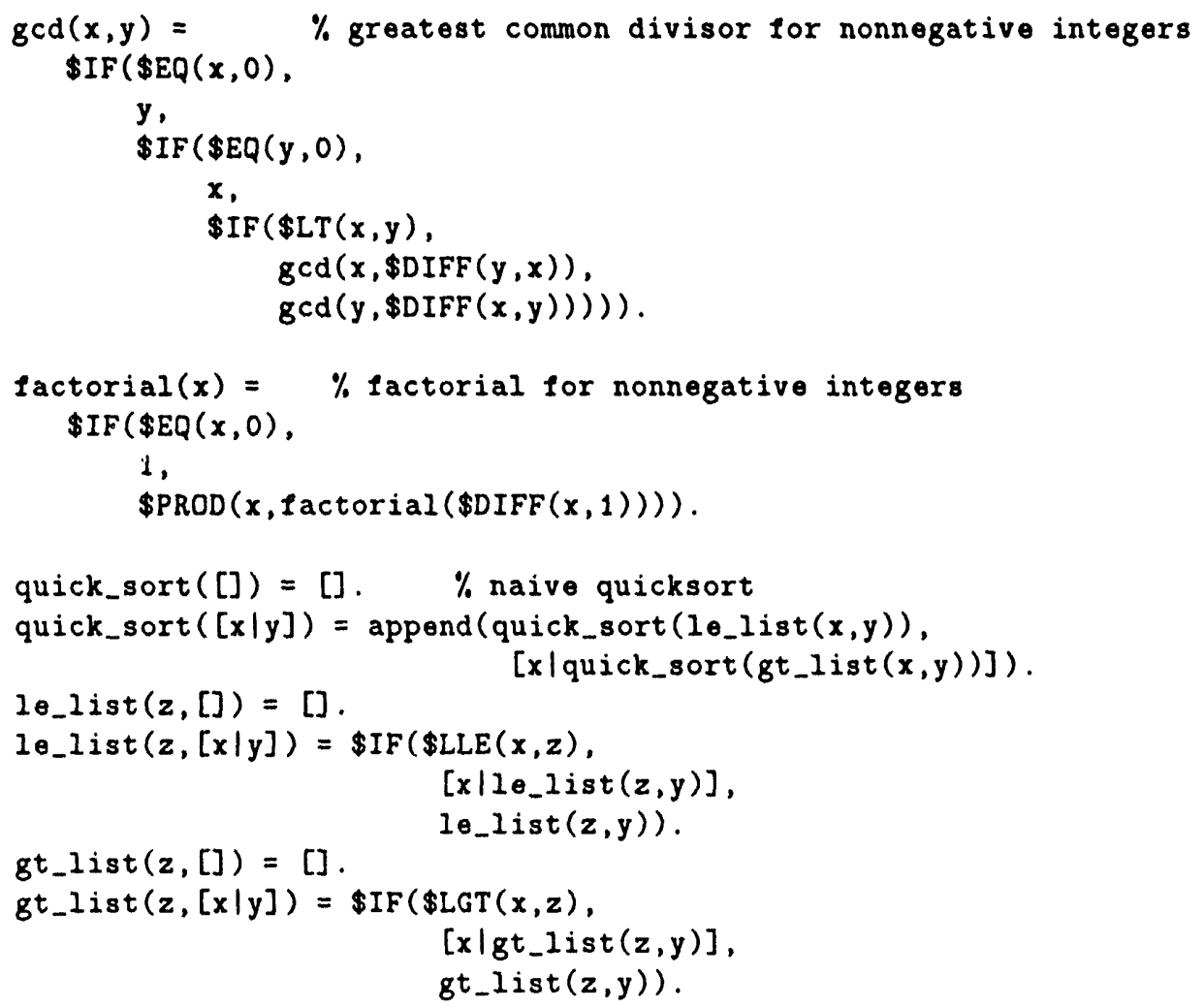

A State-Space Search. Here is a complete OTTER input file for a simple statespace search.

\% We have a 3-gallon jug and a 4-gallon jug, both empty, and a well. 


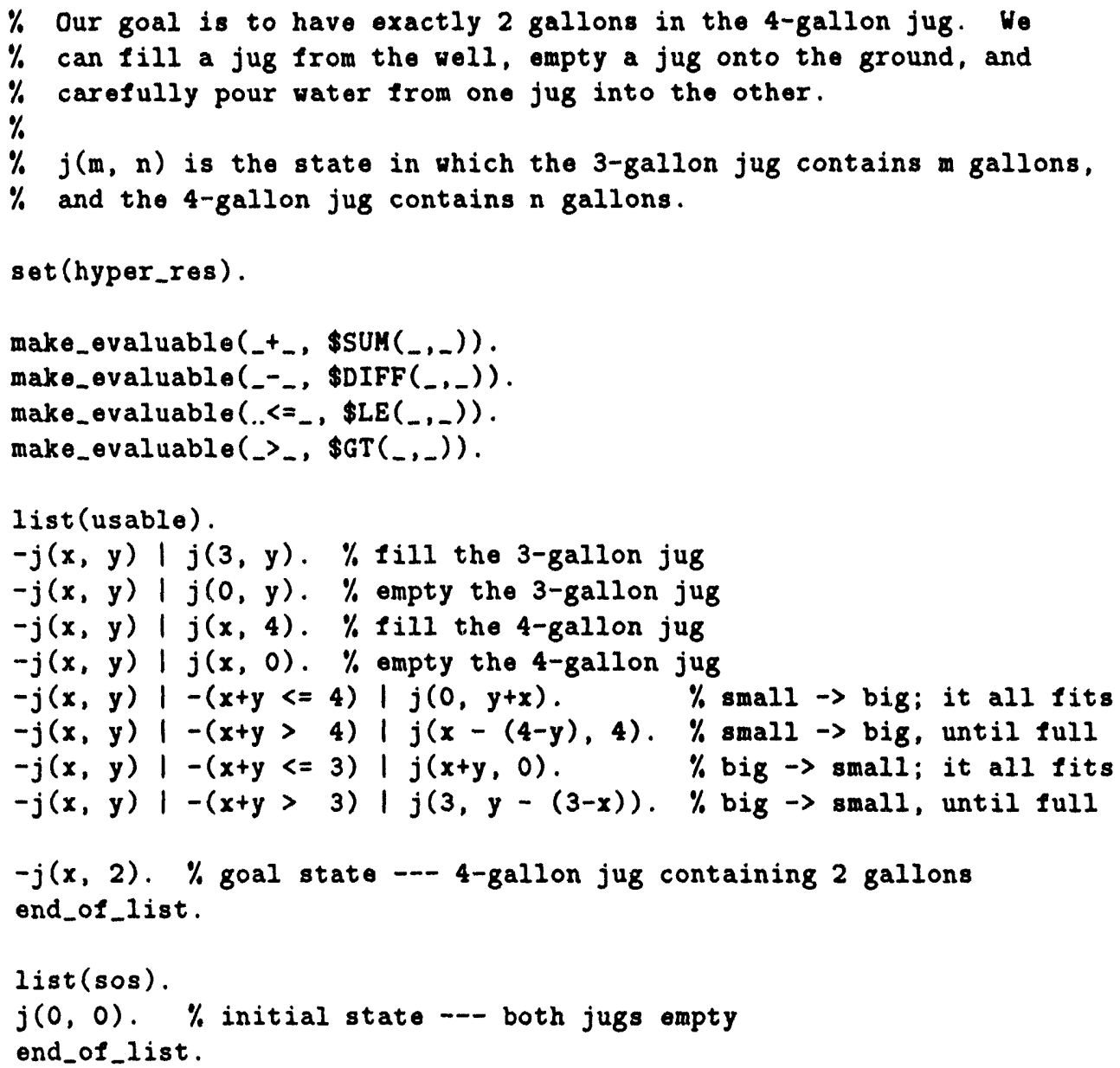

\section{Weighting}

OTTER recognizes four lists of weight templates. (See Sec. 5.4 for input of weight template lists.)

weight_list (pick_given). This list is used for selection of given clauses from list sos. When the weight of a clause is printed, it is the pick_given weight.

weight_list(purge_gen). This list is used in conjunction with the max weight parameter to discard generated clauses.

weight_list (pick_and_purge). In many cases, one can use the same weighting strategy for both selecting given clauses and purging generated clauses. The pick_and_purge list serves the purposes of both the pick_given and the purge_gen lists. If the pick_and_purge list is present, then neither the pick_given nor the purge_gen list may be present.

weight.list(terms). This list is for calculating the weight of terms when using the weight-lex-order (Sec. 8.1.1) to compare terms. This occurs when the flag Irpo is clear when orienting equality literals (Secs. 8.1.2 and 8.1.3). 


\subsection{Weighing Clauses and Literals}

The weight of a clause is always the sum of the weights of its literals (excluding any answer literals). The weight of a positive literal is the weight of its atom. The weight of a negative literal is the weight of its atom plus the value of the neg-weight parameter (Sec. 6.2.5).

\subsection{Weighing Atoms and Terms}

Atoms and terms are weighed top-down. To weigh a given term, OTTER searches the appropriate weight list (in the order input) for the first matching template. If a match is found, then the subterms of the given term that match the integers in the template are weighed. The weight of the given term is the sum of the products of each integer and the weight of its corresponding subterm, plus the second argument of the weight template. For example, the template

$$
\text { weight }(f(g(\$(2)), \$(-3)),-50) \text {. }
$$

matches the given term

$$
f(g(h(a)), f(b, x)) \text {. }
$$

Let $w t(t)$ be the wejght of term or atom $t$. Then

$$
w t(f(g(h(a)), f(b, x)))=2 * w t(h(a))+(-3) * w t(f(b, x))+(-50)
$$

If a matching weight template is not found, then the weight of the given term is 1 plus the sum of the weights of the subterms. (See the flags atom_wt_max_args and term_wt_max_args, Sec. 6.1.9, for overrides.) Note that this weighting scheme implies that if no weight templates are present, the default weight of a term or atom is the number of variable, constant, function, and predicate symbols (the symbol count).

Variables in weiglit templates are generic. A variable in a weight template will match any variable, and only a variable, in the given term. As a consequence, it is never necessary to use different variable names in a weight template. For exarnple, weight $(f(x, x),-7)$ matches the term $f(u, v)$, and weight $(x, 32)$ matches all variables.

Warning. The two occurrences of symbol $f$ in the term $f(f, x)$ are treated by OTTER as different symbols because they have different arities. The weight template weight $(f, 0)$ applies to the second occurrence but not to the first.

The default weight of an answer literal is 0, but templates can be used to assign weights to answer literals. The parameter neg_weight never applies to answer literals.

If one wishes to have a weight template containing a Skolem function or constant that is generated by OTTER, one must first make a short trial run to find out how 
the formulas are Skolemized, then return to the input file and insert the weight list containing the Skolem symbol after the formula lists.

\section{Answer Literals}

The main use of answer literals is to record, during a search for a refutation, instantiations of variables in input clauses. For example, if the theorem under consideration states that an object exists, then the denial of the theorem contains a variable, and an answer literal containing the variable can be appended to the denial. If a refutation is found, then the empty clause has an answer literal that contains the object whose existence has just been proved.

Any literal whose predicate symbol starts with \$ans, \$Ans, or \$ANS is an answer literal. Most routines--including the ones that count literals and decide whether a clause is positive or negative-ignore any answer literals. The inference rules insert, into the children, the appropriate instances of any answer literals in the parents. If factoring is enabled, OTTER does attempt to factor answer literals.

\section{The Passive List}

Either clauses or formulas can be input to list passive. After input, the passive list is fixed for the rest of the run. Clauses in the passive list are used for exactly two purposes: forward subsumption and unit conflict. If forward subsumption is enabled, a newly generated clause will be deleted if it is subsumed by any clause in usable, sos, or passive, and newly kept unit clauses are checked for unit conflict against unit clauses in usable, sos, or passive.

The passive list has been most useful for monitoring the progress of a search. Suppose we are trying to prove a difficult theorem, we have some lemmas in mind, and we would like to know whether OTTER has proved the lemmas. Then denials of the lemmas can be placed in the passive list, and OTTER will report proofs if it proves any lemmas, but the denials of the lemmas will not interfere with the search for the main theorem. (Recall that an appropriate value must be assigned to max_proofs; otherwise OTTER will stop at the first proof.)

\section{Completeness and Soundness}

\subsection{Completeness}

If the clause set does not involve equality, or if it involves equality and includes the equality axioms, then many of the common refutation-complete resolution search strategies can be easily achieved with OTTER. For example, hyperresolution and factoring, with positive clauses in the list sos and nonpositive clauses in the list usable, is complete. If the input clause set is IIorn, then factoring is not required. 
The default method of sclecting the given clause (take one with the fewest symbols) does not interfere with completeness, and neither forward nor back subsumption, as implemented in OTTER, interferes with completeness of the basic inference rules.

Completeness issues are more complex when paramodulation is the inference rule, especially when the set of support strategy is considered. A simple and complete paramodulation strategy for OTTER is (1) paramodulate from and into the given clause, (2) paramodulate from and into both sides of equality literals, (3) paramodulate from (but not into) variables, and (4) place all input clauses in the list sos. The equality $x=x$ is required, but the functionally reflexive axioms are not required.

Compieteness of the basic inference rules is important, but incomplete restrictions and refinements are frequently required to find proofs. For example, I almost always use the max_weight parameter; strictly speaking, it is incomplete, but it saves a lot of time and memory, and careful use of it does not prevent OTTER from finding proofs in practice. For paramodulation, I generally use a search based on some variation of the Knuth-Bendix completion procedure; some versions are known to be incomplete, and others have not been analyzed. I sometimes use UR-resolution on nonHorn sets, which is incomplete. And I make extensive use of weighting to purge "uninteresting clauses" and the options delete_identical_nested_skolem, max_distinct_vars, and max_literals, all of which interfere with completeness.

\subsection{Soundness}

As far as I know, no part of OTTER has been formally verified in any way. If it finds a proof, it can print the proof line by line (excluding individual demodulation steps), so the user has the option of checking it. If anything depends on the proof, I recommend at least scanning the proof for obvious errors. The few soundness bugs in previous versions of OTTER have surfaced in ways that are easy to spot in proofs, for example, deriving $x=y$ from a nontrivial equational theory.

I won't jump off a bridge (even a small one) if someone finds a bug that makes OTTER unsound, but I will tell everyone about the the bug and try to fix it promptly.

\section{Interaction during the Search}

OTTER has a primitive interactive feature that allows the user to interrupt the search, modify the options, and then continue the search. The interrupt is triggered in two ways: (1) with OTTER running in the foreground, the user types the "interrupt" character (often DELETE or ccitrol-C), or (2) if the parameter interrupt_given is set to $n$, the search is interrupted after every $n$ given clauses. When interrupted, OTTER immediately goes into a simple loop to read and execute commands. The accepted commands are listed in Table 8.

The following notes elaborate on the interactive feature.

- The flag interactive_given (Sec. 6.1.1) can be useful with the interactive 
Table 8: Interaction Commands

\begin{tabular}{ll}
\hline help. & Give simple help. \\
set (flag-name). & Set a flag. \\
clear(flag-name). & Clear a flag. \\
assign(param-name, value) & Assign a value to a parameter. \\
stats. & Send statistics to std. output and the terminal. \\
usable. & Print list usable on the terminal. \\
sos. & Print list sos on the terminal. \\
demodulators. & Print list demodulators on the terninal. \\
passive. & Print list passive on the terminal. \\
fork. & Fork and run the child process; \\
& resume parent when child finishes. \\
continue. & Continue the search. \\
kill. & Send statistics to standard output, and exit.
\end{tabular}

feature. For example, if the user thinks the search is going to fail, he or she can interrupt it, print list sos, set the interactive_given flag, then continue, selecting given clauses interactively.

- The fork command creates a separate copy, called a child, of the entire OTTER process. Immediately after the fork, the child is running (waiting for more commands) and the original process, the parent, is waiting for the child to finish. When the child finishes, the parent resumes (waiting for more commands). Changes that the child makes to the clause space, options, etc., are not reflected in the parent; when the parent resumes, it is in exactly the same state as when the fork occurred. (The timing statistics are not handled correctly in child processes; CPU times are from the start of the current process; wall-clock time is correct; other timings are not reliable.)

- The interactive routine is an area where a user who is also a $\mathrm{C}$ programmer can easily add features. For example, most of the ordinary input commands could be made available in the interactive mode.

Warning. Do not interactively change any option that affects term or literal indexing.

\section{Output and Exit Codes}

OTTER sends most of its output to "standard output", which is usually redirected by the user to a file; I'll just call it the output file. The first part of the output file is an echo of most of the input and some additional information, including identification numbers for clauses and description of some input processing. Comments are not echoed to the output. The second part of the output file reflects the search. Various print flags determine what is output. Given clauses, generated clauses, kept clauses, and several messages about the processing of generated and kept clauses can be printed. Both statistics from the parameter report and proofs can also be printed 
during the search. The final part of the output file lists counts of various events (such as clauses given and clauses kept) and times for various operations.

Whenever a clause is printed, it is printed with its integer identifier (ID) and a justification list, which is enclosed in brackets. Examples:

4[]$-j(x, y) \mid j(x, 0)$.

13 [hyper, 11,8, eval, demod] $j(3,1)$.

$41[31$, demod] $p([a, b, b, c, c, c, d, \theta, f])$.

14 [new_demod, 13] $f(y, f(y, f(y, x)))=x$.

71 [back_demod, 58 , demod, $70,14,55,11,34,11]$ ] $!=\theta$.

$12[$ demod, 9$] \quad f(a, f(b, f(g(a), g(b)))) !=\theta$.

77 [binary $57.3,30.2] \mathrm{sm} / \mathrm{mm} /-81$.

33,32 [para_from, 26.1.1,15.1.1.2, demod, 21] $g(x)=f(x, x)$.

36 [hyper, $31,2,26,30$, unit_del $19,18,20,19] \mathrm{p}(\mathrm{k}, \mathrm{g}(\mathrm{k}))$.

4 [factor_simp, factor_simp] $p(x)|p(\$ f 1(x))|-q(\$ f 2(y))|-q(y)| p(\$ c 6)$. 199 [binary, 198.1,191.1, factor_simp] $q(\$ c 14)$.

If the justification list is empty, the clause was input. Otherw ${ }^{\circ}$, the first item in the justification list is one of the following.

An inference rule. The clause was generated by an inference rule. The IDs of the parents are listed after the inference rule with the given clause ID listed first (unless order_history is set).

A clause identifier. The clause was generated by the demod_inf rule.

new_demod. The clause is a dynamically generated demodulator; it is a copy of the clause whose ID is listed after new_demod.

back_demod. The clause was generated by back demodulating the clause whose ID is listed after back_demod.

demod. The clause was generated by back demodulating an input clause.

factor_simp. The clause was generated by factor-simplifying an input clause. For example, $p(x) \mid p(a)$ factor-simplifies to $p(a)$.

The sublist [demod, $i d_{1}, i d_{2}, \ldots$ ] indicates demodulation with $i d_{1}, i d_{2}, \ldots$. The sublist [unit_del, $i d_{1}, i d_{2}, \ldots$ ] indicates unit deletion with $i d_{1}, i d_{2}, \ldots$. The symbols oval indicates that a literal was "resolved" by evaluation (Sec. 9) during hyperresolution. The sublist [factor simp, factor simp,...] indicates a sequence of factorsimplification steps (Sec. 6.1.4).

In proofs, some clauses are printed with two (consecutive) IDs. In such a case, the clause is a dynamically generated demodulator, and the two IDs refer to different copies of the same clause: the first ID refers to its use for inference rules, and the second to its use as a demodulator.

If the flag detailed_history is set, then for the inference rules binary_res, para_from, and para_into, the positions of the unified literals or terms are listed 
along with the parent IDs. For example, [binary , 57.3,30.2] means that the third literal of clause 57 was resolved with the second literal of clause 30 . For paramodulation, the "from" parent is listed as ID.i.j, where $i$ is the literal number of the equality literal, and $j$ (either 1 or 2 ) is the number of the unified equality argument; the "into" parent is listed as ID.i. $j_{1}, \cdots, j_{n}$, where $i$ is the literal number of the "into" term, and $j_{1}, \cdots, j_{n}$ is the position vector of the "into" term; for example, 400.3.1.2 refers to the second argument of the first argument of third literal of clause 400 . If the flag para_all is set, then the paramodulation positions are not listed.

When the flag sos_queue is set, the search is breadth first (level saturation), and OTTER sends a message to the output file when given clauses start on a new level. (Input clauses have level 0 , and generated clauses have level one greater than the maximum of the levels of the parents. Since clauses are given in the order in which they are retained, the level of given clauses never decreases.)

Exit Codes. When OTTER stops running, it returns with an exit code that gives the reason for termination. The codes are useful when another program or system calls OTTER. Table 9 lists the exit codes. Note that we do not follow the UNIX convention of returning zero for normal and nonzero for abnormal termination.

\begin{tabular}{ll}
\multicolumn{1}{c}{ Table 9: Exit Codes } \\
\hline 101 & Input error(s) \\
102 & Abnormal end (compile-time limit or OTTER bug) \\
103 & Proof(s) found (stopped by max_proofs) \\
104 & sos list empty \\
105 & max_given parameter exceeded \\
106 & max_seconds parameter exceeded \\
107 & max_gen parameter exceeded \\
108 & max_kept parameter exceeded \\
109 & max_mem parameter exceeded \\
110 & Operating system out of memory \\
111 & Interactive exit \\
112 & Memory error (probable OTTER bug) \\
\hline
\end{tabular}

\section{Controlling Memory}

In many OTTER searches, the sos list accumulates many clauses that never enter the search, possibly wasting a lot of memory. The normal way to conserve memory is to put a maximum on the weight of kept clauses. It can be difficult, however, to find an appropriate maximum. OTTER has a feature, enabled by the command set(control_memory), that attempts to automatically adjust the maximum.

The memory-control feature operates as follows. When one third of available memory (max_mem parameter) has been filled, OTTER assigns or reassigns a maximum weight. The new maximum, say $n$, is such that $5 \%$ of all clauses in sos have 
weight $\leq n$. From then on, at every tenth iteration of the main loop, OTTER calculates a prospective new maximum $n^{\prime}$ in the same way. If $n^{\prime}<n$, then the maximum is reset to $n^{\prime}$. I arrived at the values $1 / 3$ and $5 \%$ by trial and error. Perhaps these values should be parameters.

\section{Fringe Features}

This section describes some features that are new, not well tested, and/or not well documented.

\subsection{Autonomous Mode}

If the flag auto is set, OTTER will scan the input clauses for some simple syntactic properties and decide on inference rules and a search strategy. We think of the autonomous mode as providing a built-in metastrategy for selecting search strategies. The search strategy that OTTER selects for a particular set of clauses is usually refutation complete (except for the flag control_memory), $L$, t the user should not expect it to be especially effective. It will find proofs for many easy theorems, and even for cases in which it fails to find a proof, it provides a reasonable starting point.

In the input file, the command set (auto) must occur before any input clauses, and all input clauses must be in list usable; it is an error to place input clauses on any of the other lists when in autonomous mode. OTTER will move some of the input clauses to sos before starting the search. When OTTER processes the set (auto) command, it alters some options, even before examining the input clauses. If the user wishes to augment the autonomous mode by including some ordinary OTTER commands (including overriding OTTER's choices), the commands should be placed after set (auto) and before list (usable).

After list (usable) has been read, OTTER examines the input clauses for several syntactic properties and decides which inference rules and strategies should be used, and which clauses should be moved to sos. The user cannot override the decisions that OTTER makes at this stage.

OTTER looks for the following syntactic properties of the set of input clauses: (1) whether it is propositional, (2) whether it is Horn, (3) whether equality is present, (4) whether equality axioms are present, and (5) the maximum number of literals in a clause. The program then considers six basic combinations of the properties: (1) propositional, (2) equality in which all clauses are units, and (3-6) the four combinations of \{equality, Horn\}. To see precisely what OTTER does for these cases, the reader can set up and run some simple experiments.

Please be aware that the autonomous mode reflects my own experiences with OTTER; other users would certainly formulate different metastrategies. For example, Larry Wos prefers UR-resolution to hyperresolution or in addition to hyperresolution in rich Horn or nearly-llorn theories, and he prefers to add few or no dynamic demodulators for equality theories. 


\subsection{The Hot List}

The hot list is a strategy that can be used to emphasize particular clauses. It was invented by Larry Wos in the context of paramodulation, and it has been extended so most of OTTER's inference rules. To use the strategy, the user simply inputs one or more clauses in the special list named hot. Whenever a clause is generated and kept by O'TTER's ordinary mechanisms, it is immediately considered for inference with clauses in the hot list.

Which Clauses Should Be Hot? Clauses input in the hot list are usually copies of clauses that occur also in sos or usable. They are usually clauses that the user believes will play a key role in the search for a proof, for example, the special hypothesis.

Managing Hot-List Clauses. Input to the hot list is the same as input to other lists and can be in either clause or formula form, for example,

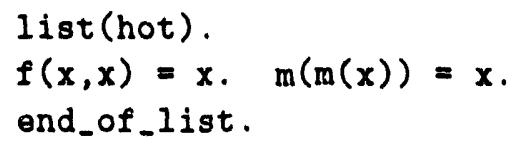

The flag process_input has no effect on hot-list clauses; they are never altered during input. Hot-list clauses are never deleted, for example by back subsumption or back demodulation. Even if a hot-list clause is identical to a clause in another list, it has a unique identifying number, and proofs that use hot-list clauses generally refer to two copies (with different ID numbers) of those clauses.

Hot Inference Rules. The inference rules that are applied to newly kept clauses and hot-list clauses are the same as the rules in effect for ordinary inference, with the exceptions demod_inf, geometric_rule, and linked_ur_res, which are never applied to hot-list clauses.

Applying Hot Inference. When hot inference is applied, the newly kept clause is treated as the given clause, and the hot list is treated as the usable list. (Note that the newly kept clause is not in the hot list, so it will not be considered for inference with itself, as happens with the given clause in ordinary inference.) For inference rules such as hyperresolution or UR-resolution that can use more than two parents, all of the other parents must be in the hot list; this generally means that the nucleus and other satellites must be in the hot list. Hot inference is not applied to clauses that are "kept" during processing of the input.

Level of Hot Inference (Parameter heat). To prevent long sequences of hot inferences (i.e., hot inference applied to a clause generated by hot inference, and so on) we consider the heat level of hot inference. The heat level of an ordinary 
inference is 0 , and the heat level of a hotly inferred clause is one more than the heat level of the rew-clause parent. The parameter heat, default 1 , range $[0 . .100]$, is the maximum heat level that will be generated. When a clause is printed, its heat level, if greater than 0 , is also printed.

Dynamic Hot Clauses (Parameter dynamic_hoat_woight). Clauses can be added to the hot list during a search. If the pick_given weight of a kept clause is less than or equal to the parameter dynamic_heat_weight, default -MAX_INT, range $[-M A X\lrcorner N T . . M A X\lrcorner N T]$, then the clause will be added to the hot list and used for subsequent hot inference. Input clauses that are "kept" during processing of the input are never made into dynamic hot clauses. Dynamic hot clauses can be added to an empty hot list (i.e., no input hot list).

\subsection{Linked UR-Resolution}

OTtEr has an inference rule, linked_ur_res, that is an application of the linked inference principle [27] to UR-resolution. As this manual is written, there is not yet any documentation. The inference rule is still evolving and is highly experimental. For current information on the status of linked UR-resolution, send e-mail to wos@mcs.anl.gov and veroff@cs.unm.edu.

\subsection{Conditional Demodulation}

A conditional demodulator has the form

condition $\rightarrow$ equality-literal.

The equality is applied as a demodulator if and only if the instantiated condition evaluates to $\$ r$. The equality of a conditional denodulator is not subjected on input to being flipped or to being flagged as a lex-dependent demodulator, and conditional demodulators are never back demodulated. In other ways, conditional demodulators behave as ordinary demodulators. Examples are (member and ged are defined in Sec. 9.)

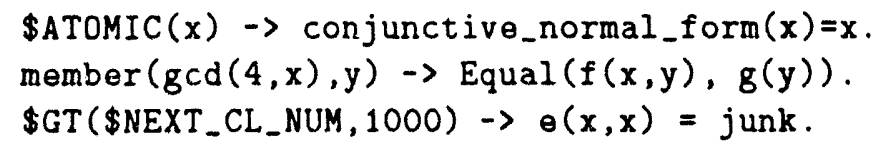

\subsection{Special Unary Function Demodulation}

A feature, activated by the special_unary command, allows OTTER to avoid one of the problems caused by the lack of associative-commutative matching during demodulation. The feature is useful when an associative-commutative function and an inverse are present, as in rings. Without this feature, the following lex command and demodulators 


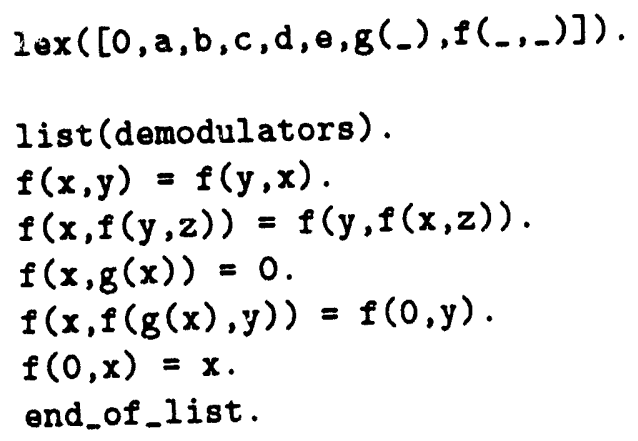

will cause the expression

$$
f(f(f(g(b), a), c), f(b, g(c)))
$$

to be sorted into

$$
f(a, f(b, f(c, f(g(b), g(c))))) \text {. }
$$

One would like $b$ and $g(b)$ to be next to each other so that they could be canceled by one of the inverse demodulators. The special-unary feature accomplishes just that. The command

$$
\text { special_unary }(g(x)])
$$

causes $\mathrm{g}$ to be ignored during term comparisons, and the expression would be demodulated to a. The special_unary command has no effect if the flag Irpo is set. This is an experimental fcature. Its behavior has not been well analyzed.

\subsection{Ancestor Subsumption}

OTTER does not necessarily prefer short or simple proofs-it simply reports the proofs that it finds. An option ancestor_subsume extends the concept of subsumption to include the derivation history, so that if two clause occurrences are logically identical, the one with fewer ancestors is preferred. The motivation is to find short proofs.

ancestor_subsume - default clear. If this flag is set, the notion of subsumption (forward and back) is replaced with ancestor-subsumption. Clause $C$ ancestorsubsumes clause $D$ iff $C$ properly subsumes $D$ or if $C$ and $D$ are variants and size (ancestorset $(C)) \leq$ size(ancestorset $(D))$.

When setting ancestor_subsume, we strongly recommend not clearing the flag back_subsume, because doing so can cause many occurrences of the same clause to be retained and used as given clauses. 


\subsection{Reducing max_weight on the Fly}

In many searches, the number of kept clauses grows much faster than the number of given clauses. In other words, the list sos is very large, and most of those clauses never participate in the search. To save memory, one can use the max_weight parameter to discard many of the clauses that will (probably) never become given clauses.

A few searches and proofs show a phenomenon we call the complexity hump. To get a search started, one must use complex clauses; then one can continue the search using simpler clauses. That is, the first few steps in the proof are complex, and the remaining steps are simpler. If one needs to carefully conserve memory when a complexity hump is present, one can use the parameters change_limit_after and new_max_weight to change the value of max_weight after a specified number of given clauses.

change_limit_after - default 0 , range [0..MAXJNT]. If $n$ (the value) is not 0 , this parameter has effect. After $n$ given clauses have been used, the parameter max_weight is automatically reset to the value of the parameter new_max_weight.

new_max_weight - default MAX_INT, range [-MAX_INT..MAX_JNT]. See the description of the preceding parameter.

Note that the memory-control feature (Sec. 16) can also address the complexity hump phenomenon.

\subsection{The Invisible Argument}

OTTER recognizes a built-in unary function symbol \$IGNORE(_). Forward subsumption treats each term that starts with \$IGNORE as the constant \$IGNORE, completely ignoring its argument. For example, $p(a, \$ \operatorname{IGNORE}(b))$ subsumes $p(a, \$ \operatorname{IGNORE}(c))$. All other operations (in particular, inference rules, demodulation, and back subsumption) treat \$IGNORE as an ordinary function symbol.

The purpose of \$IGNORE is to record data about the derivation of a clause without having that data prevent the forward subsumption of clauses that would be subsumed without that data. The \$IGNORE term is the term analog of the answer literal. For example, one can use \$IGNORE terms in the jugs and water puzzle (Sec. 9.2 ) to record the sequence of pourings that leads to each state.

\subsection{Floating-Point Operations}

Table 10 lists a set of floating-point evaluable functions and predicates that are analogous to the integer arithmetic operations listed in Sec. 9. They operate in the same way as the integer operations.

The floating-point constants, however, are a little peculiar, both in the way they look and in the way they behave. They are written as quoted strings, using either single or double quotes. (Otherwise, they would not be able to contain decimal 
Table 10: Floating-Point Operations

\begin{tabular}{|l|l|}
\hline float $\times$ float $\rightarrow$ float & \$FSUM, \$FPROD, \$FDIFF, \$FDIV, \$FMOD \\
\hline float $\times$ float $\rightarrow$ bool & \$FEQ, \$FNE, \$FLT, \$FLE, \$FGT, \$FGE \\
\hline
\end{tabular}

points.) Other than the quotation marks, the form of the floating-point constants accepted by OTTER is exactly the same as the form accepted by the $\mathrm{C}$ programming language (actually the C library used by the compiler). Examples are "1.2", "1006", "-3.333E-5". A floating-point constant must contain either a decimal point or an exponent character $\Theta$ or $E$.

The peculiar behavior comes from the fact OTTER stores the floating point numbers as character strings instead of directly as floating point numbers. To apply a floating-point operation, OTTER starts with the operand strings, translates them to true floating-point numbers (the $\mathrm{C}$ data type "double" is used), performs the operation, then translates the result into a string so that it can be an OTTER constant. As well as being inefficient, this scheme also has a problem with precision, because a fixed format is used to translate the results back into strings. The default format is "\%.12f", and it can be changed with a command such as

float_format ("\%17.8f")

Caution. Otter does not check that the string in the float_format command is a well-formed format specification. This is the user's responsibility.

To fully understand how this works, see the standard $\mathrm{C}$ language reference $[11$, Appendix B]; in particular, the $C$ library functions sscanf and sprintf are used to translate to and from strings.

\subsection{Foreign Evaluable Functions}

OTTER provides a general mechanism through which the user can create his or her own evaluable functions and predicates. The user (1) declares the function, its argument types, and its result type, (2) inserts a call to the function in the OTTER source code, (3) writes a $\mathrm{C}$ routine to implement the function, and (4) recompiles OTter. The user must have his or her own copy of the source code to use this feature. See the source code file foreign.h for step-by-step instructions, examples, templates, and test files.

Important note. Many times you can avoid having to do all of this by just writing your function with demodulators and using existing built-in functions. For example, if you need the maximum of two doubles, you can just use the demodulator float_max $(x, y)=\$ I F(\$ F G T(x, y), x, y)$.

\subsection{Sequent Notation for Clauses}

There are two flags that enable the use of sequent notation for clauses. 
input_sequent - default clear. If this flag is set, clauses in the input file must be in sequent notation.

output_sequent - default clear. If this flag is set, then sequent notation is used when clauses are output.

Syntax:

- All sequent clauses have an arrow.

- The negative literals (if any) are written on the left side of the arrow, are written without the negation sign, and are separated by commas.

- The positive literals (if any) are written on the right side of the arrow and are separated by commas.

Table 11 lists some examples.

Table 11: Examples of Sequent Clauses

\begin{tabular}{l|l}
\hline Ordinary Clause & Sequent Clause \\
\hline$-p|-q|-r|s| t \mid$ & $p, q, r->s, t$ \\
$p(a, b, c)$ & $\rightarrow p(a, b, c)$ \\
$a !=b$ & $a=b \rightarrow$ \\
$\$ F$ (the empty clause) & $\rightarrow>$ \\
\hline
\end{tabular}

Note that $\mathrm{p}, \mathrm{q}->\mathrm{r}, \mathrm{s}$ is ordinarily thought of as ( $\mathrm{p}$ and $\mathrm{q}$ ) implies ( $\mathrm{r}$ or $\mathrm{s}$ ).

Sequent clauses are treated as (parsed as) a special case, because they can't be made to fit within OTTER's ordinary syntax.

\subsection{The Inference Rule $g L$ for Cubic Curves}

Based on work of R. Padmanabhan and others, a new inference rule, $g L$ ("geometric Law", or "Local to global"), was added to OTTER. The rule implements a local-to-global generalization principle that has a geometric interpretation for cubic curves. The article [18] contains a description of the rule, some details about its implementation in OTTER, and several new results obtained with its use.

The rule $g L$ applies to single positive unit equalities, and it is implemented in two ways: as an inference rule, with unification, and as a rewrite rule, for when the target ierms are already identical. The following flags, usually used together, enable the rule.

geometric_rule - default clear. When this flag is set, $g L$ is applied as an inference rule (along with any other inference rules that are set) to each given clause. The rule $g L$ applies to single positive unit equalities.

geometric_rewrite - default clear. When this flag is set, $g L$ is applied as a rewrite rule, after ordinary demodulation, to each generated clause. 
Our experience has shown that given two equalities of equal weight, one the result of $g L$ and the other not, the $g L$ result is usually more interesting. The following parameter can give preference to $g L$ results.

geo_given_ratio - default 1 , range $[-1$..MAX_JNT]. When this parameter is not -1 , it affects selection of the given clause in a way similar to pick_given_ratio. If the ratio is $n$, then for each $n$ given clauses selected in the normal way by weight, one given clause is selected because it is the lightest $g L$ result available in sos. If pick_given_ratio and geo_given_ratio are both in effect, then clashes are resolved in favor of geo_given_ratio.

\section{Limits, Abnormal Ends, and Fixes}

OTTER has several compile-time limits. If a limit is exceeded, a message containing the name of the limit will appear in the output file and/or at the terminal. To raise the limit, find the appropriate definition (\#define) in a .h or .c file, increase the limit, and recompile OTTER. (Of course, one must have his or her own copy of the source code to do this.) Some of the limits are as follows.

MAX_NAME - Maximum number of characters in a variable, constant, function, or predicate symbol.

MAX_EUF Maximum number of characters in an input string (clause, formula, command, weight template, etc.).

MAX_VARS - Maximum number of distinct variables in a clause.

MAX_FS_TERM_DEPTH - Maximum depth of terms in the forward subsumption discrimination tree.

MAX_AL_TERM_DEPTH - Maximum depth of left-hand arguments of equalities in the demodulation discrimination tree.

Conserving Memory. Several steps can be taken if OTTER is using too much memory.

- Use max_weight to discard (morc) generated clauses. This is a very effective way to save memory (and time).

- Set the flag control_memory (Sec. 6.1.9), or use the parameters change_limit_after and new_max_weight (Sec. 17.7).

- Decrease (down to 0) the value of the fpa_literals and fpa_terms parameters.

- Set the for_sub_fpa flag to switch forward subsumption indexing from discrimination tree to FPA indexing.

- If the inference rules being used are binary resolution or paramodulation, clear the flag detailed_history. 
- If a lot of back subsumption or back demodulation is expected, set the flag really_delete_clauses (Sec. 6.1.9).

- If applicable, set no_fapl or no_fanl (Sec. 6.1.8).

- If back demodulation is being used, clear the flag index_for_back_demod.

- Run an OTter job until memory runs out, collect interesting lemmas from the output file, then rerun the job including the lemmas as input clauses. Repeat. (This can be a good strategy even when memory is not a problem.)

\section{Obtaining and Installing OTTER}

OTTER 3 is free, and there are no restrictions on copying or distributing it. The main means of distribution is anonymous FTP from info.mcs.anl.gov. See the file README in the directory pub/Otter for information on the current state and versions of OTTER 3.

Once you have a copy of the OTTER 3 distribution directory, you can compile OTTER. (There may be Macintosh and DOS binaries available; see below.) The directory source contains all of the source code and a UNIX-style makefile. On many UNIX-like operating systems, including Linux 99.pl13, SunOS 4.1.3, AIX 3.2.2, NeX'TStep 3.1, and IRIX 4.0.5, simply typing "make otter" should compile OTTER. If compilation fails, see comments in the file makefile for hints on getting OTTER. to compile on your system.

Once you have OTTER compiled, go to the directory test and see the file README. You can then run the test and example input files in that directory.

As I write this manual, OTTER 3 has not been compiled for DOS or Macintosh computers; I hope that those versions will soon become available in binary as well as in source form. Inquiries on OTrER 3 for DOS or Macintosh systems can be sent to the autlior.

\section{Acknowledgments}

A side from writing and maintaining OTrer, much of my work over the past few years has been in collaboration with Larry Wos. Toward our goal of creating programs that are expert assistants for mathematicians, logicians, engineers, and other scientists, we have worked together on many applications of automated deduction, and that work has led to many of OTTER's current features.

The basic design of the program, including the data structures and the use of indexing, descends mostly from early programs of Ross Overbeek. The indexing mechanisms, which are in large part responsible for the performance of the program, have benefited from discussions with Overbeek, Mark Stickel, and Rusty Lusk.

The expert users of O'Trer, including Bob Veroff, Ken Kunen, John Kalman, and Art Quaife, have tracked down bugs and suggested useful culancements. 


\section{References}

[1] W. Bledsoc and D. Loveland, editors. Automated Theorem Proving: After 25 Years, volume 29 of Contemporary Mathematics. AMS, 1984.

[2] R. S. Boyer and J S. Moore. A Computational Logic. Academic Press, New York, 1979.

[3] R. S. Boyer and J S. Moore. A Computational Logic Handbook. Academic Press, New York, 1988.

[4] C.- L. Chang and R. C.-T. Lee. Symbolic Logic and Mechanical Theorem Proving. Academic Press, New York, 1973.

[5] N. Dershowitz. Termination of rewriting. Journal of Symbolic Computation, 3:69-116, 1987.

[6] C. A. R. Iloarc and M. J. C. Gordon, editors. Mechanized Reasoning and Hardware Design. Prentice-IIall, 1992.

[7] J S. Moore, editor. Special Issue on System Verification. Journal of Automated Reasoning, 5(4), 1989.

[8] J.-P. Jouannaud, editor. Rewriting Techniques and Applications, Lecture Notes in Computer Scicnce, Vol. 202, New York, 1985. Springer-Verlag.

[9] D. Kapur, editor. Proceedings of the 11th International Conference on Automatcd Deduction, Lecture Notes in Artificial Intelligence, Vol. 607, New York, 1992. Springer-Verlag.

[10] D. Kapur and II. Zhang. RRL: Rewrite rule laboratory user's manual. Technical Report 89-03, Department of Computer Science, University of Iowa, 1989.

[11] B. Kernighan and D. Ritchie. The C Programming Language. Prentice-Hall, 2nd ed., 1988.

[12] D. Knuth and P. Bendix. Simple word problems in universal algebras. In J. Leech, editor, Computational Problems in Abstract Algebras, pages 263-297. Pergamon Press, Oxford, U.K., 1970.

[13] A. G. Kurosh. The Theory of Groups, volume 1. Chelsea, New York, 1956.

[14] D. Loveland. Aulomated Theorem Proving: A Logical Basis. North-Holland, Amsterdam, 1978.

[15] E. Lusk and R. Ovcrbeek. The automated reasoning system ITP. Tech. Report ANL-84/27, Argonne National Laboratory, Argonne, Ill., April 1984.

[16] W. McCune. Skolem functions and equality in automated deduction. In Procecdings of the Eighth National Conference on Artificial Intelligence, pages 246251, Cambridge, Mass., 1990. MIT Press.

[17] W. McClune. Single axioms for groups and Abelian groups with various operations. Journal of Automated Reasoning, 10(1):1-13, 1993. 
[18] R. Padmanabhan and W. McCune. Automated reasoning about cubic curves. Computers and Mathematics with Applications, 1993. To appear.

[19] A. Quaife. Automated development of Tarski's geometry. Journal of Automated Reasoning, 5(1):97-118, 1989.

[20] A. Quaife. Automated Development of Fundamental Mathematical Theories. Ph.D. thesis, University of California at Berkeley, 1990.

[21] J. Siekmann and G. Wrightson, editors. Auomation of Reasoning: Classical Papcrs on Computational Logic, volume 1 and 2. Springer-Verlag, Berlin, 1983.

[22] B. Smith. Reference manual for the environmental theorem prover: An incarnation of AURA. Tech. Report ANL-88-2, Argonne National Laboratory, Argonne, Ill., March 1988.

[23] M. Stickel, editor. Proccedings of the 10th Intcrnational Conference on Automated Deduction, Lecture Notes in Artificial Intelligence, Vol. 199 , New York, 1990. Springer-Verlag.

[24] L. Wos. Automated Reasoning: 33 Basic Research Problems. Prentice-Iall, Englewood Cliffs, N.J., 1988.

[25] L. Wos, R. Overbeek, E. Lusk, and J. Boyle. Automated Reasoning: Introduction and Applications, revised edition. McGraw-Hill, New York, 1992.

[26] L. Wos, F. Pereira, R. Boyer, J Moore, W. Bledsoe, L. Henschen, B. Buchanan, G. Wrightson, and C. Green. An overview of automated reasoning and related fields. Journal of Automated Reasoning, 1(1):5-48, 1985.

[27] L. Wos, R. Veroff, B. Smith, and W. McCune. The linked inference principle II: The user's view. In R. Shostak, editor, Proceedings of the 7th International Conference on Automated Deduction, Lecture Notes in Computer Science, Vol. 170, pages 316-332, New York, 1981. Springer-Verlag. 


\section{Distribution for ANL-94/6}

Internal:

J. M. Beumer (125)

F. Y. Fradin

E. L. Lusk

W. W. McCune (50)

R. A. Overbeek

G. W. Piepe:

R. L. Stevens

C. L. Wilkinson

L. T. Wos

TIS File

External:

DOE-OSTI, for distribution per UC-405 (54)

ANL-E Library (2)

ANL-W Library

Manager, Chicago Operations Office, DOE

Mathematics and Computer Science Division Review Committee:

W. W. Bledsoe, The University of Texas, Austin

B. L. Buzbee, National Center for Atmospheric Research

J. G. Glimm, State University of New York at Stony Brook

M. T. Heath, University of Illinois, Urbana

E. F. Infante, University of Minnesota

D. O'Leary, University of Maryland

R. E. O'Malley, Rensselaer Polytechnic Institute

M. H. Schultz, Yale University

J. Cavallini, Department of Energy - Office of Scientific Computing

F. Howes, Department of Energy - Office of Scientific Computing

J. Kalman, The University of Auckland

$K$. Kunen, University of Wisconsin at Madison

A. Quaife, Trans Time, Inc., Dakland, CA

M. Stickel, SRI International, Palo Alto, CA

$R$. Veroff, University of New Mexico 

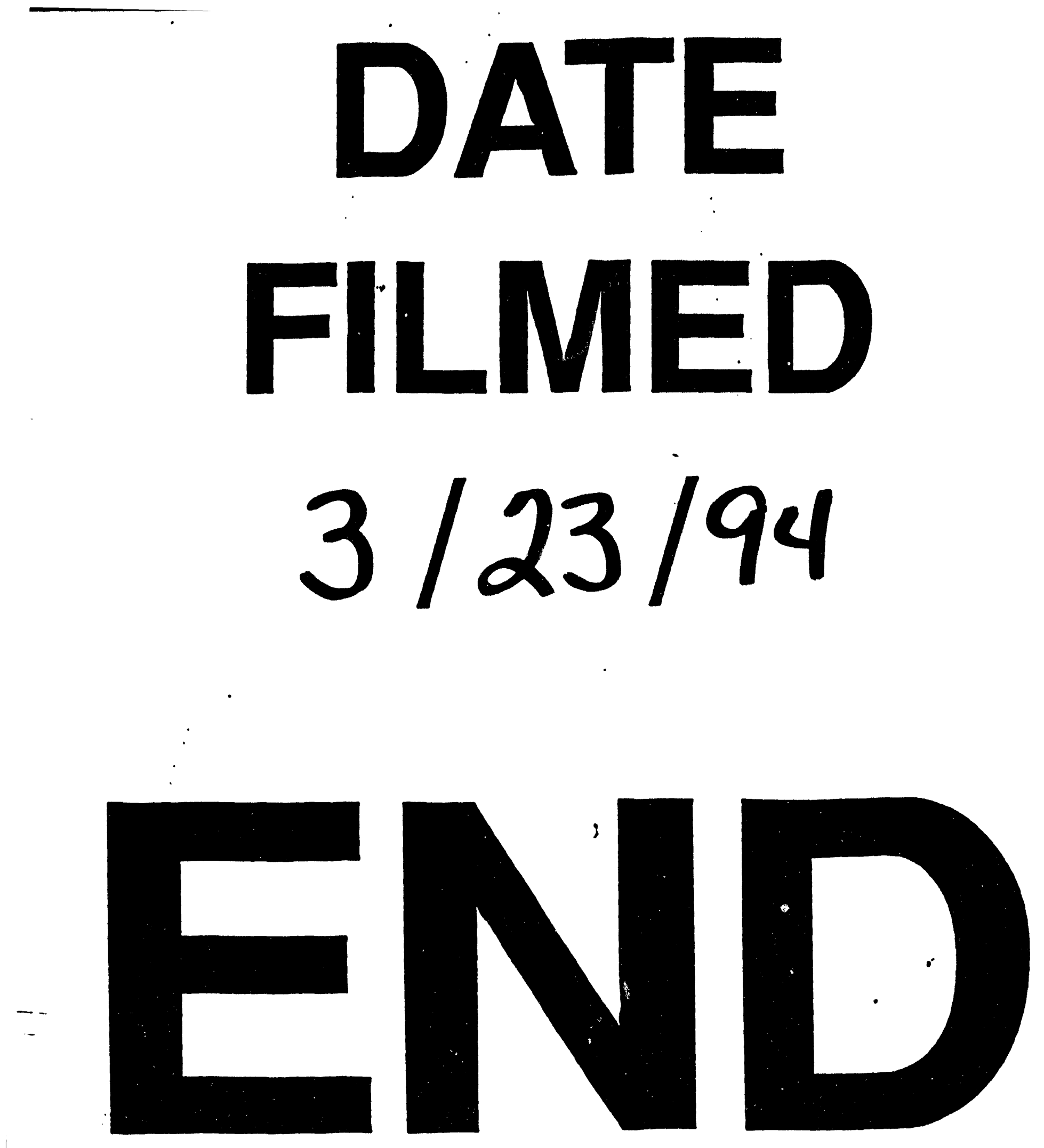
